CARPATHIAN J. MATH.

Volume 38 (2022), No. 1,

Pages $95-116$
Online version at https : //www . carpathian. cunbm. utcluj. ro/

Print Edition: ISSN 1584 - 2851; Online Edition: ISSN 1843 - 4401

DOI: https://doi.org/10.37193/CJM.2022.01.08

Dedicated to the memory of Academician Mitrofan M. Choban (1942-2021)

\title{
Well-posedness of a nonlinear second-order anisotropic reaction-diffusion problem with nonlinear and inhomogeneous dynamic boundary conditions
}

\author{
Mitrofan M. ChOBAn and Costică N. MoroşAnU
}

ABSTRACT. The paper is concerned with a qualitative analysis for a nonlinear second-order boundary value problem, endowed with nonlinear and inhomogeneous dynamic boundary conditions, extending the types of bounday conditions already studied. Under certain assumptions on the input data: $f_{1}(t, x), w(t, x)$ and $u_{0}(x)$, we prove the well-posedness (the existence, a priori estimates, regularity and uniqueness) of a classical solution in the Sobolev space $W_{p}^{1,2}(Q)$. This extends previous works concerned with nonlinear dynamic boundary conditions, allowing to the present mathematical model to better approximate the real physical phenomena (the anisotropy effects, phase change in $\Omega$ and at the boundary $\partial \Omega$, etc.).

\section{INTRODUCTION}

For the unknown function $u(t, x)$ (hereafter, $u$ ), consider the following nonlinear secondorder parabolic problem in $Q=(0, T] \times \Omega$, with $T>0$ and a bounded domain $\Omega \subset \mathbb{R}^{n}$, $n \in\{1,2,3\}$ of Lebesgue measures $|\Omega|$, whose boundary $\partial \Omega=\Gamma$ is sufficiently smooth

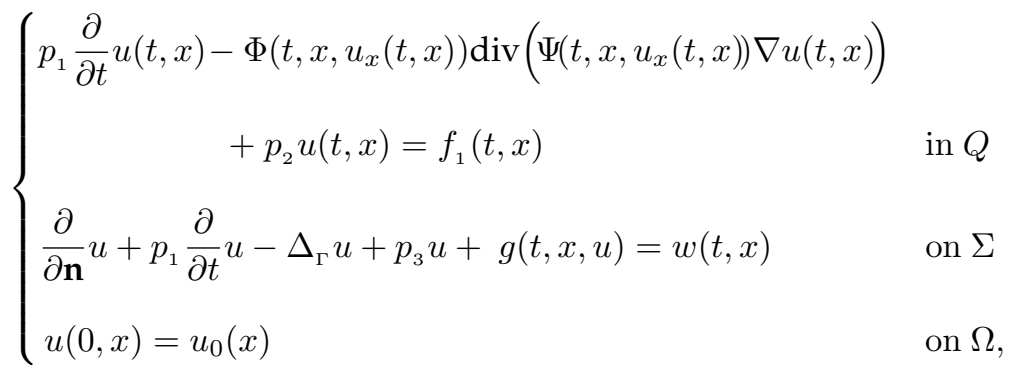

where:

- $t \in(0, T], x=\left(x_{1}, \ldots, x_{n}\right) \in \Omega, \Sigma=(0, T] \times \Gamma$;

- $u(t, x)$ is the unknown function and $\nabla u(t, x)=u_{x}(t, x)\left(\nabla u=u_{x}\right.$ in short) is the gradient of $u(t, x)$ in $x$, that is

$$
\nabla u=\left(\frac{\partial}{\partial x_{1}} u, \frac{\partial}{\partial x_{2}} u, \cdots, \frac{\partial}{\partial x_{n}} u\right) \text {. We set } \frac{\partial}{\partial x_{i}} u=u_{x_{i}}, i=1,2, \ldots, n \text {, and so }
$$
$u_{x}=\left(u_{x_{1}}, u_{x_{2}}, \cdots, u_{x_{n}}\right)$;

- $\frac{\partial}{\partial t} u(t, x)$ is the partial derivative of $u(t, x)$ with respect to $t$;

- $p_{1}, p_{2}, p_{3}$ are positive values;

Received: 22.02.2021. In revised form: 08.09.2021. Accepted: 15.09.2021

2010 Mathematics Subject Classification. 35Bxx, 35K55, 35K60, 35Qxx.

Key words and phrases. nonlinear second-order anisotropic reaction-diffusion prblem, qualitative properties of solutions, Leray-Schauder principle, nonlinear inhomogeneous dynamic boundary conditions.

Corresponding author: Costică Moroşanu; costica.morosanu@uaic.ro 
- $\left.\Phi\left(t, x, u_{x}(t, x)\right)\right)$ - is a positive and bounded nonlinear real function of class $C^{1}(Q)$ with bounded derivatives, having the role of controlling the speed of the diffusion process;

- $\Psi\left(t, x, u_{x}(t, x)\right)$ - is the non-constant mobility (attached to the solution $u(t, x)$ of (1.1));

- $f_{1}(t, x) \in L^{p}(Q)$ is the distributed control (a given function), where

$$
p \geq 2
$$

- $\mathbf{n}=\mathbf{n}(\mathbf{x})$ is the outward unit normal vector to $\Omega$ at a point $x \in \partial \Omega$. $\frac{\partial}{\partial \mathbf{n}}$ denotes differentiation along $\mathbf{n}$;

- $\Delta_{\Gamma}$ is the Laplace-Beltrami operator;

- $g: \Sigma \times \mathbb{R} \rightarrow \mathbb{R}$ is a Carathéodory function, that is, $g(\cdot, \cdot, z): \Sigma \rightarrow \mathbb{R}$ is measurable, $\forall z \in \mathbb{R}$, and $g(t, x, \cdot): \mathbb{R} \rightarrow \mathbb{R}$ is continuous, $\forall(t, x) \in \Sigma$, with $g(\cdot, \cdot, 0) \in L^{\infty}(\Sigma)$ (see [12] and reference therein). Moreover, the following hypotheses are assumed to be satisfied:

$$
\begin{aligned}
\mathbf{g}_{1}: & \left(g\left(t, x, z_{1}\right)-g\left(t, x, z_{2}\right)\right)\left(z_{1}-z_{2}\right) \geq b_{1}\left(z_{1}-z_{2}\right)^{2}, \quad \forall(t, x) \in \Sigma, \\
& z_{1}, z_{2} \in \mathbb{R}, \text { for a constant } b_{1}>0 ;
\end{aligned}
$$

$\mathbf{g}_{2}$ : there is a function $\bar{G}: \Sigma \times \mathbb{R}^{2} \rightarrow \mathbb{R}$ verifying the relations

$$
\begin{aligned}
& \left(g\left(t, x, z_{1}\right)-g\left(t, x, z_{2}\right)\right)^{2} \leq \bar{G}\left(t, x, z_{1}, z_{2}\right)\left(z_{1}-z_{2}\right)^{2}, \\
& \bar{G}\left(t, x, z_{1}, z_{2}\right) \leq b_{2}\left(1+\left|z_{1}\right|^{2\left(r^{\prime}-1\right)}+\left|z_{2}\right|^{2\left(r^{\prime}-1\right)}\right), \quad \forall(t, x) \in \Sigma, \\
& z_{1}, z_{2} \in \mathbb{R}, \text { for a constant } b_{2}>0 \text { and } r^{\prime} \geq 1 \text { such that } \\
& \text { (see relation (3.19) below) }
\end{aligned}
$$

$$
\frac{n+2}{n+2-2 p} \geq r^{\prime} \quad \text { if } \frac{1}{p}-\frac{2}{n+2}>0
$$

$\mathbf{g}_{3}: g(t, x, z) z \geq b_{3} z^{2}, \quad \forall(t, x) \in \Sigma, z \in \mathbb{R}$, with $b_{3}>0$.

- $w(t, x) \in W_{p}^{1-\frac{1}{2 p}, 2-\frac{1}{p}}(\Sigma)$ is the boundary control (a given function);

- $u_{0}(x) \in W_{\infty}^{2-\frac{2}{p}}(\Omega)$, verifying

$$
\frac{\partial}{\partial \mathbf{n}} u_{0}-\Delta_{\Gamma} u_{0}+p_{3} u_{0}+g\left(0, x, u_{0}\right)=w(0, x) .
$$

Lemma 1.1. Assumption $\mathbf{g}_{2}$ implies that $g(t, x, z)$ fulfills the polynomial growth condition

$$
|g(t, x, z)| \leq b_{4}\left(1+|z|^{r^{\prime}}\right), \quad \forall(t, x) \in \Sigma, \quad z \in \mathbb{R},
$$

where $b_{4}$ is a positive constant.

Proof. Indeed, setting $z_{1}=z$ and $z_{2}=0$ in $\mathbf{g}_{2}$, we get

$$
\begin{gathered}
|g(t, x, z)| \leq|g(t, x, 0)|+\bar{G}(t, x, z, 0)^{\frac{1}{2}}|z| \\
\leq|g(t, x, 0)|+b_{2}^{\frac{1}{2}}\left(1+|z|^{2\left(r^{\prime}-1\right)}\right)^{\frac{1}{2}}|z|, \quad \forall z \in \mathbb{R} .
\end{gathered}
$$

Since $g(t, x, 0) \in L^{\infty}(\Sigma)$, estimate (1.4) follows. 
For reader's benefit, we will write problem (1.1) in the equivalent form

$$
\left\{\begin{array}{lc}
p_{1} \frac{\partial}{\partial t} u(t, x)-\Phi\left(t, x, u_{x}(t, x)\right) \frac{\partial}{\partial u_{x_{j}}}\left(\Psi\left(t, x, u_{x}(t, x)\right) u_{x_{i}}\right) u_{x_{j} x_{i}} & \\
=A\left(t, x, u_{x_{i}}\right)-p_{2} u(t, x)+f_{1}(t, x) & \text { in } Q \\
\frac{\partial}{\partial \mathbf{n}} u+p_{1} \frac{\partial}{\partial t} u-\Delta_{\Gamma} u+p_{3} u+g(t, x, u)=w(t, x) & \text { on } \Sigma \\
u(0, x)=u_{0}(x) & \text { on } \Omega
\end{array}\right.
$$

with

$$
A\left(t, x, u_{x_{i}}\right)=\Phi\left(t, x, u_{x}(t, x)\right) \frac{\partial}{\partial x_{i}}\left(\Psi\left(t, x, u_{x}(t, x)\right) u_{x_{i}}\right)
$$

and

$$
u_{x_{i}}=\frac{\partial}{\partial x_{i}} u(t, x), \quad u_{x_{j} x_{i}}=\frac{\partial^{2}}{\partial x_{j} \partial x_{i}} u(t, x), \quad i, j=1, \ldots, n .
$$

It is easy to recognize (1.5) as being a quasi-linear one of type (2.4) in [4, p. 3 and p. 11], with

and

$$
a_{i j}\left(t, x, u_{x}(t, x)\right)=\frac{\partial}{\partial u_{x_{j}}} \Psi\left(t, x, u_{x}(t, x)\right) u_{x_{i}}, i=1, \ldots, n,
$$

$$
a\left(t, x, u(t, x), u_{x}(t, x)\right)=-A\left(t, x, u_{x}(t, x)\right)+p_{2} u(t, x)-f(t, x),
$$

while the boundary conditions (1.5) $)_{2}$ are of second type, with

$$
\frac{\partial}{\partial \mathbf{n}} u(t, x)=a_{i j}\left(t, x, u, u_{x}\right) u_{x_{j}} \cos \alpha_{i},
$$

$\alpha_{i}$ being the angle formed by the outward unit normal vector $\mathbf{n}(\mathbf{x})$ to $\Omega$ at $x \in \partial \Omega$ with the $x_{i}$ axis, and

$$
\left.\psi(t, x, u)\right|_{\Sigma}=p_{1} \frac{\partial}{\partial t} u-\Delta_{\Gamma} u+p_{3} u+g(t, x, u)-w(t, x)
$$

(see [4, p. 475, relation (7.2)]).

In addition, unless otherwise stated, we assume that equations (1.1) $)_{1}$ [or (1.5) $)_{1}$ are uniformly parabolic, which means fulfilment of the conditions

$$
\nu_{1}(|u|) \zeta^{2} \leq \frac{\partial}{\partial z_{j}} \Psi(t, x, z) \zeta_{i} \zeta_{j} \leq \nu_{2}(|u|) \zeta^{2}
$$

for arbitrary $u$ and $z$ and $\zeta=\left(\zeta_{1}, \ldots, \zeta_{n}\right)$ an arbitrary real vector, where $\nu_{1}(s)$ and $\nu_{2}(s)$ are positive (nonincreasing and nondecreasing, respectively) continuous functions of $s \geq 0$.

In the present paper we study the solvability of the nonlinear second-order boundary value problems of the form (1.1) or (1.5) in the class $W_{p}^{1,2}(Q)$. The new mathematical formulation expressed by (1.1) is characterized by the presence of some new physical parameters: $\left.p_{1}, p_{2}, p_{3}, \Phi\left(t, x, u_{x}(t, x)\right), \Psi\left(t, x, u_{x}(t, x)\right)\right)$, the principal part being in divergence form and by considering a linear reaction term (see [1]). The most important novelty in our paper concerns the inhomogeneous dynamic boundary conditions of nonlinear type, untreated until now (in this new context) in the mathematical literature. Thus, significant aspects of the delicate physical features are expected to be reflected more accurately. In this regard, as applications of problem (1.1) or (1.5), we indicate the moving interface problems, e.g. phase separation and transition (see [2], [6], [8], [12], [13]), anisotropy effects (see [1], [7]), image denoising and segmentation (see [1], [7] and references therein), etc. 


\section{PRELIMINARIES AND MAIN RESUlT}

In order to approach the parabolic nonlinear problem (1.1), we will use the same idea as in [2], [6], [8] [9], [10] and [11]. In this respects, let $\zeta=u$ be a further variables such that $\zeta(0, x)=u_{0}(x)$ on $\Gamma$ (see also [4, p. 449, relation (6.2)]), while for the remaining data in (1.1) we will keep the same meanings already formulated in introduction. Corresponding, the boundary conditions in $(1.1)_{2}$ will be approached in the following by

$$
\begin{cases}u(t, x)=\zeta(t, x) & \text { on } \Sigma \\ \frac{\partial}{\partial \nu} u+p_{1} \frac{\partial}{\partial t} \zeta-\Delta_{\Gamma} \zeta+p_{3} \zeta+g(t, x, \zeta)=w(t, x) & \text { on } \Sigma \\ \zeta(0, x)=\zeta_{0}(x) & x \in \Gamma,\end{cases}
$$

where $\zeta_{0}(x) \in W_{\infty}^{2-\frac{2}{p}}(\Gamma)$. Accordingly, the nonlinear second-order boundary value problem (1.5) can be rewritten suitably as follows

$$
\left\{\begin{array}{lc}
p_{1} \frac{\partial}{\partial t} u(t, x)-\Phi\left(t, x, u_{x}(t, x)\right) \frac{\partial}{\partial u_{x_{j}}}\left(\Psi\left(t, x, u_{x}(t, x)\right) u_{x_{i}}\right) u_{x_{j} x_{i}} & \\
=A\left(t, x, u_{x_{i}}\right)-p_{2} u(t, x)+f_{1}(t, x) & \text { in } Q \\
u(t, x)=\zeta(t, x) & \text { on } \Sigma \\
\frac{\partial}{\partial \mathbf{n}} u+p_{1} \frac{\partial}{\partial t} \zeta-\Delta_{\Gamma} \zeta+p_{3} \zeta+g(t, x, \zeta)=w(t, x) & \text { on } \Sigma \\
u(0, x)=u_{0}(x) & \text { on } \Omega \\
\zeta(0, x)=\zeta_{0}(x) & x \in \Gamma,
\end{array}\right.
$$

where $u_{0}(x)=\zeta_{0}(x)$ on $\Gamma$ and $\zeta_{0}(x) \in W_{\infty}^{2-\frac{2}{p}}(\Gamma)$.

Definition 2.1. Any solution $(u(t, x), \zeta(t, x))$ of the nonlinear second-order boundary value problem (2.10) is called the classical solution if it is continuous in $\bar{Q}$, have continuous derivatives $u_{t}, u_{x}, u_{x x}$ in $Q$ and $\zeta_{t}, \zeta_{x}, \zeta_{x x}$ on $\Sigma$, satisfy the equation (2.10) $)_{1}$ at all points $(t, x) \in Q$ and satisfy conditions (2.10) ${ }_{2-3}$ and (2.10) $4-5$ on the lateral surface $\Sigma$ of the cylinder $Q$ and for $t=0$, respectively.

Our main results regarding the existence, uniqueness and regularity of solutions to problem (2.10) (practically, well-posedness of the solutions to the nonlinear second-order boundary value problem (1.1) or (1.5)) is the following

Theorem 2.1. Suppose $(u(t, x), \zeta(t, x)) \in C^{1,2}(Q) \times C^{1,2}(\Sigma)$ is a classical solution of problem (2.10) and for positive numbers $M, M_{0}, m_{1}, M_{1}, M_{2}, M_{3}, M_{4}$ and $M_{5}$ one has

$\mathbf{I}_{1} .|u(t, x)|<M$ for any $(t, x) \in Q$ and for any $z(t, x)$, the map $\psi(t, x, z)$ is continuous, differentiable in $x$, its $x$-derivatives are measurable bounded, satisfies (1.8), and

$$
0<\Psi_{\min } \leq \Psi\left(t, x, u_{x}(t, x)\right)<\Psi_{\max }, \quad \text { for }(t, x) \in Q
$$




$$
\begin{aligned}
& \sum_{i=1}^{n}\left|\Psi(t, x, z) u_{x_{i}}\right|(1+|z|) \\
& +\sum_{i, j=1}^{n}\left|\frac{\partial}{\partial x_{j}}\left(\Psi(t, x, z) u_{x_{i}}\right)\right|+|u(t, x)| \leq M_{0}(1+|z|)^{2} .
\end{aligned}
$$

$\mathbf{I}_{2} . \Phi\left(t, x, u_{x}(t, x)\right)$ is a positive and bounded nonlinear real function of class $C^{1}(Q)$ with bounded derivatives and

$$
m_{1} \leq \Phi\left(t, x, u_{x}(t, x)\right) \leq M_{1} .
$$

In addition, for any sufficiently small $\varepsilon>0$, the functions $u(t, x)$ and $\Psi\left(t, x, u_{x}(t, x)\right)$ satisfy the relations

where

$$
\|u\|_{L^{s}(Q)} \leq M_{2}, \quad\left\|\Psi\left(t, x, u_{x}(t, x)\right) u_{x_{i}}\right\|_{L^{r}(Q)}<M_{3}, \quad i=1, \ldots, n,
$$

$$
r=\left\{\begin{array}{ll}
\max \{p, 4\} & p \neq 4 \\
4+\varepsilon & p=4,
\end{array} \quad s= \begin{cases}\max \{p, 2\} & p \neq 2 \\
2+\varepsilon & p=2\end{cases}\right.
$$

$\mathbf{I}_{3}$. The hypotheses $\mathbf{g}_{1}-\mathbf{g}_{3}$ are fulfilled.

Then, $\forall f_{1} \in L^{p}(Q), u_{0} \in W_{\infty}^{2-\frac{2}{p}}(\Omega), \zeta_{0}(x) \in W_{\infty}^{2-\frac{2}{p}}(\Gamma), w \in W_{p}^{1-\frac{1}{2 p}, 2-\frac{1}{p}}(\Sigma)$, with $p \neq \frac{3}{2}$, there exists a unique solution $(u, \zeta) \in W_{p}^{1,2}(Q) \times W_{p}^{1,2}(\Sigma)$ to (2.10) and satisfies

$$
\begin{aligned}
\|u\|_{W_{p}^{1,2}(Q)}+\|\zeta\|_{W_{p}^{1,2}(\Sigma)} & \leq C\left[1+\left\|u_{0}\right\|_{W_{\infty}^{2-\frac{2}{p}}(\Omega)}+\left\|\zeta_{0}\right\|_{W_{\infty}^{2-\frac{2}{p}}(\Gamma)}\right. \\
+ & \left.\left\|f_{1}\right\|_{L^{p}(Q)}+\|w\|_{L^{p}(\Sigma)}+\|w\|_{W_{p}^{1-\frac{1}{2 p}, 2-\frac{1}{p}}(\Sigma)}\right]
\end{aligned}
$$

where the constant $C>0$ is independent of $u, \zeta, f_{1}$ and $w$.

If $\left(u^{1}, \zeta^{1}\right),\left(u^{2}, \zeta^{2}\right)$ are two solutions to $(2.10)$ corresponding to $\left(u_{0}^{1}, \zeta_{0}^{1}\right),\left(u_{0}^{2}, \zeta_{0}^{2}\right) \in W_{\infty}^{2-\frac{2}{p}}(\Omega) \times$ $W_{\infty}^{2-\frac{2}{p}}(\Gamma), f_{1}^{1}, f_{1}^{2}, w^{1}$ and $w^{2}$, respectively, such that

$$
\begin{array}{cl}
\left\|u^{1}\right\|_{W_{p}^{1,2}(Q)}, & \left\|u^{2}\right\|_{W_{p}^{1,2}(Q)} \leq M_{4}, \\
\left\|\zeta^{1}\right\|_{W_{p}^{1,2}(\Sigma)}, & \left\|\zeta^{2}\right\|_{W_{p}^{1,2}(\Sigma)} \leq M_{5},
\end{array}
$$

then the following estimate holds

$$
\begin{aligned}
\max _{(t, x) \in Q}\left|u^{1}-u^{2}\right|+\max _{(t, x) \in \Sigma}\left|\zeta^{1}-\zeta^{2}\right| & \\
\leq & C_{1} e^{C T} \max \left\{\max _{(t, x) \in \Omega}\left|u_{0}^{1}-u_{0}^{2}\right|, \underset{(t, x) \in \Gamma}{\max }\left|\zeta_{0}^{1}-\zeta_{0}^{2}\right|,\right. \\
& \left.\max _{(t, x) \in Q}\left|f_{1}^{1}-f_{1}^{2}\right|, \max _{(t, x) \in \Sigma}\left|w^{1}-w^{2}\right|\right\},
\end{aligned}
$$

where the positive constants $C_{1}>0, C>0$, are independent of $\left\{u^{1}, \zeta^{1}, f_{1}^{1}, w^{1}, u_{0}^{1}, \zeta_{0}^{1}\right\}$ and $\left\{u^{2}, \zeta^{2}, f_{1}^{2}, w^{2}, u_{0}^{2}, \zeta_{0}^{2}\right\}$. In particular, the uniqueness of solution to problem (2.10) holds.

As far as the techniques used in the paper are concerned, it should be noted that we derive the a priori estimates in $L^{p}(Q)$ and $L^{p}(\Sigma)$. Moreover, basic tools in our approach are: 
- the Leray-Schauder degree theory (see [8, p. 221] and reference therein);

- the $L^{p}$-theory of linear and quasi-linear parabolic equations [4];

- Green's first identity

$$
\begin{gathered}
-\int_{\Omega} y \operatorname{div} z d x=\int_{\Omega} \nabla y \cdot z d x-\int_{\partial \Omega} y \frac{\partial}{\partial \mathbf{n}} z d \gamma, \\
-\int_{\Omega} y \Delta z d x=\int_{\Omega} \nabla y \cdot \nabla z d x-\int_{\partial \Omega} y \frac{\partial}{\partial \mathbf{n}} z d \gamma,
\end{gathered}
$$

for any scalar-valued function $y$ and $z$, a continuously differentiable vector field in $n$ dimensional space;

- the Lions and Peetre embedding Theorem [5, p. 24] to ensure the existence of a continuous embedding $W_{p}^{1,2}(Q) \subset L^{\mu}(Q)$, where the number $\mu$ is defined as follows (see $(1.2))$

$$
\mu= \begin{cases}\infty & \text { if } \frac{1}{p}-\frac{2}{n+2}<0, \\ \text { any number } \geq p & \text { if } \frac{1}{p}-\frac{2}{n+2}=0, \\ \frac{p(n+2)}{n+2-2 p} & \text { if } \frac{1}{p}-\frac{2}{n+2}>0 .\end{cases}
$$

For a given positive integer $k$ and $1 \leq p \leq \infty$, we denote by $W_{p}^{k, 2 k}(Q)$ the Sobolev space on $Q$ :

$$
W_{p}^{k, 2 k}(Q)=\left\{y \in L^{p}(Q): \frac{\partial^{i}}{\partial t^{i}} \frac{\partial^{j}}{\partial x^{j}} y \in L^{p}(Q), \text { for } 2 i+j \leq 2 k\right\},
$$

i.e., the spaces of functions whose $t$-derivatives and $x$-derivatives up to the order $k$ and $2 k$, respectively, belong to $L^{p}(Q)$. Also, we will use the Sobolev spaces $W_{p}^{i}(\Omega), W_{p}^{\frac{i}{2}, i}(\Sigma)$ with nonintegral $i$ for the initial and boundary conditions, respectively (see [4, p. 70 and p. 81]).

Also, we shall use the set $C^{1,2}(\bar{D})\left(C^{1,2}(D)\right)$ of all continuous functions in $\bar{D}$ (in $D$ ) having continuous derivatives $u_{t}, u_{x}, u_{x x}$ in $\bar{D}$ (in $\left.D\right)(D=Q$ or $D=\Sigma$ ), as well as the Sobolev spaces $W_{p}^{l}(\Omega), W_{p}^{l, l / 2}(\Sigma)$ with nonintegral $l$ for the initial and boundary conditions, respectively (see $[4$, p. 8, p. 70 and p. 81]).

In the following we will denote by $C$ several positive constants, being understood that the extra dependencies will be set out on occurrence.

\section{Proof of the MAIN RESUlt - Theorem 2.1}

To prove this theorem, we use the Leray-Schauder principle. Let $p^{\prime}$ chosen as follows

$$
\mu \geq p^{\prime}= \begin{cases}\text { any number } \geq p r^{\prime} & \text { if } \frac{1}{p}-\frac{2}{n+2} \leq 0, \\ \text { any number in }\left[p r^{\prime}, \frac{p(n+2)}{n+2-2 p}\right] & \text { if } \frac{1}{p}-\frac{2}{n+2}>0 .\end{cases}
$$

Notice that (3.19) makes sense due to (1.3). 
Consider the Banach space $B=W_{p}^{0,1}(Q) \times L^{p^{\prime}}(\Sigma)$ endowed with the norm $\|\cdot\|_{B}$, expresed by

$$
\|(\varphi, \bar{\varphi})\|_{B}=\|\varphi\|_{L^{p}(Q)}+\left\|\varphi_{x}\right\|_{L^{p}(Q)}+\|\bar{\varphi}\|_{L^{p^{\prime}}(\Sigma)},
$$

and a nonlinear operator $H: B \times[0,1] \rightarrow B$ defined by

$$
(u, \zeta)=H(\varphi, \bar{\varphi}, \lambda)=(u(\varphi, \bar{\varphi}, \lambda), \zeta(\varphi, \bar{\varphi}, \lambda)) \quad \forall(\varphi, \bar{\varphi}) \in B, \forall \lambda \in[0,1],
$$

where $(u(\varphi, \bar{\varphi}, \lambda), \zeta(\varphi, \bar{\varphi}, \lambda)$ is the unique solution to the following linear second-order boundary value problem

$$
\left\{\begin{array}{lc}
p_{1} \frac{\partial}{\partial t} u-\left[\lambda \Phi\left(t, x, \varphi_{x}\right) \frac{\partial}{\partial \varphi_{x_{j}}}\left(\Psi\left(t, x, \varphi_{x}\right) \varphi_{x_{i}}\right)-(1-\lambda) \delta_{i}^{j}\right] u_{x_{i} x_{j}} & \\
=\lambda\left[A\left(t, x, \varphi_{x_{i}}\right)-p_{2} \varphi(t, x)+f_{1}(t, x)\right] & \text { in } Q \\
u(t, x)=\zeta(t, x) & \text { on } \Sigma \\
u(0, x)=\lambda u_{0}(x) & \text { on } \Omega \\
\frac{\partial}{\partial \mathbf{n}} u+p_{1} \frac{\partial}{\partial t} \zeta-\Delta_{\Gamma} \zeta+p_{3} \zeta=\lambda[-g(t, x, \bar{\varphi})+w(t, x)] & \text { on } \Sigma \\
\zeta(0, x)=\lambda \zeta_{0}(x) & x \in \Gamma .
\end{array}\right.
$$

Remark 3.1. The nonlinear operator $H$ in (3.20) depends on $\lambda \in[0,1]$ and its fixed point for $\lambda=1$ are solutions of problem (3.21).

Proof. We start the proofing of Theorem 2.1 by showing that the nonlinear operator $H$ has two properties:

A. $H$ is well-defined.

B. $H$ is continuous and compact.

A. $H$ is well-defined if the problem (3.21) has a unique solution.

Making use of the assumptions $\mathrm{I}_{1}, \mathrm{I}_{2}$ and (1.6), from the right-hand side of (3.21) it follows that $\forall \varphi \in W_{p}^{0,1}(Q)$, then $A\left(t, x, \varphi_{x_{i}}\right)+p_{2} \varphi(t, x)+f_{1}(t, x) \in L^{p}(Q)$. On the other hand, according to (1.4), we have that $g(t, x, \bar{\varphi}) \in L^{\frac{p^{\prime}}{r^{\prime}}}(\Sigma)$ whenever $\bar{\varphi} \in L^{p^{\prime}}(\Sigma)$. Moreover, (1.3) implies $g(t, x, \bar{\varphi}) \in L^{p}(\Sigma)$. Applying Lemma 7.4 (see Appendix) with

$$
\begin{aligned}
& f_{3}=\lambda\left[A\left(t, x, \varphi_{x_{i}}\right)-p_{2} \varphi(t, x)+f_{1}(t, x)\right] \in L^{p}(Q) \text { and } \\
& g_{3}=\lambda[-g(t, x, \bar{\varphi})+w(t, x)] \in L^{p}(\Sigma),
\end{aligned}
$$

the solution $(u, \zeta)$ to problem (3.21) exists and is unique. Furthermore, $\forall(\varphi, \bar{\varphi}) \in B, \forall \lambda \in$ $[0,1]$,

$$
(u, \zeta)=(u(\varphi, \bar{\varphi}, \lambda), \zeta(\varphi, \bar{\varphi}, \lambda)) \in W_{p}^{1,2}(Q) \times W_{p}^{1,2}(\Sigma) .
$$

Since $\mu=\frac{p(n+2)}{n+2-2 p} \geq p$ if $\frac{1}{p}-\frac{2}{n+2}>0$ (see (2.18)), we can take $\mu>p$ in all cases required by (2.18). Consequently, we have the continuous embeddings (see [5, p. 24])

$$
\left\{\begin{array}{l}
W_{p}^{1,2}(Q) \subset W_{p}^{0,1}(Q) \subset L^{p}(Q) \\
W_{p}^{1,2}(\Sigma) \subset L^{p^{\prime}}(\Sigma) \subset L^{p}(\Sigma),
\end{array}\right.
$$


which means that $H(\varphi, \bar{\varphi}, \lambda)=(u, \zeta) \in B$ for all $(\varphi, \bar{\varphi}) \in B$ and $\forall \lambda \in[0,1]$.

B. Let us now show that $H$ is continuous and compact.

Let $\varphi^{n} \rightarrow \varphi$ in $W_{p}^{0,1}(Q), \bar{\varphi}^{n} \rightarrow \bar{\varphi}$ in $L^{p^{\prime}}(\Sigma)$ and $\lambda^{n} \rightarrow \lambda$ in $[0,1]$. Using the notation

$$
\begin{aligned}
& \left(u^{n, \lambda_{n}}, \zeta^{n, \lambda_{n}}\right)=H\left(\varphi^{n}, \bar{\varphi}^{n}, \lambda^{n}\right), \\
& \left(u^{n, \lambda}, \zeta^{n, \lambda}\right)=H\left(\varphi^{n}, \bar{\varphi}^{n}, \lambda\right), \quad\left(u^{\lambda}, \zeta^{\lambda}\right)=H(\varphi, \bar{\varphi}, \lambda),
\end{aligned}
$$

and considering the difference $H\left(\varphi^{n}, \lambda^{n}\right)-H\left(\varphi^{n}, \lambda\right)$, we obtain from (3.20) and (3.21)

$$
\left\{\begin{array}{cc}
p_{1} \frac{\partial}{\partial t}\left(u^{n, \lambda_{n}}-u^{n, \lambda}\right)-\left[\lambda \Phi\left(t, x, \varphi_{x}^{n}\right) .\right. & \\
\left.\frac{\partial}{\partial \varphi_{x_{j}}^{n}}\left(\Psi\left(t, x, \varphi_{x}^{n}\right) \varphi_{x_{i}}^{n}\right)+(1-\lambda) \delta_{i}^{j}\right]\left(u_{x_{i} x_{j}}^{n, \lambda_{n}}-u_{x_{i} x_{j}}^{n, \lambda}\right) & \\
=\left(\lambda_{n}-\lambda\right)\left\{\left[\Phi\left(t, x, \varphi_{x}^{n}\right) \frac{\partial}{\partial \varphi_{x_{j}}^{n}}\left(\Psi\left(t, x, \varphi_{x}^{n}\right) \varphi_{x_{i}}^{n}\right)-\delta_{i}^{j}\right] u_{x_{i} x_{j}}^{n, \lambda_{n}}\right. & \\
\left.\quad+A\left(t, x, \varphi_{x_{i}}^{n}\right)-p_{2} \varphi^{n}(t, x)+f_{1}(t, x)\right\} & \text { in } Q \\
\left(u^{n, \lambda_{n}}-u^{n, \lambda}\right)(t, x)=\left(\zeta^{n, \lambda_{n}}-\zeta^{n, \lambda}\right)(t, x) & \text { on } \Sigma \\
\left(u^{n, \lambda_{n}}-u^{n, \lambda}\right)(0, x)=\left(\lambda_{n}-\lambda\right) u_{0}(x) & \text { in } \Omega \\
\frac{\partial}{\partial \mathbf{n}}\left(u^{n, \lambda_{n}}-u^{n, \lambda}\right)+p_{1} \frac{\partial}{\partial t}\left(\zeta^{n, \lambda_{n}}-\zeta^{n, \lambda}\right) & \\
-\Delta_{\Gamma}\left(\zeta^{n, \lambda_{n}}-\zeta^{n, \lambda}\right)+p_{3}\left(\zeta^{n, \lambda_{n}}-\zeta^{n, \lambda}\right) & \text { on } \Sigma \\
=\left(\lambda_{n}-\lambda\right)\left[-g\left(t, x, \bar{\varphi}^{n}\right)+w(t, x)\right] & \text { in } \Gamma . \\
\left(\zeta^{n, \lambda_{n}}-\zeta^{n, \lambda}\right)(0, x)=\left(\lambda_{n}-\lambda\right) \zeta_{0}(x) &
\end{array}\right.
$$

The right-hand side in (3.24) 1 belongs to $L^{p}(Q)$, since $u^{n, \lambda_{n}} \in W_{p}^{1,2}(Q)$. The embeddings $W_{\infty}^{2-\frac{2}{p}}(\Omega) \subset W_{p}^{2-\frac{2}{p}}(\Omega)$ and $W_{\infty}^{2-\frac{2}{p}}(\Sigma) \subset W_{p}^{2-\frac{2}{p}}(\Sigma)$ allows us to apply Lemma 7.4 with:

$f_{3}=\left(\lambda_{n}-\lambda\right)\left[A\left(t, x, \varphi_{x_{i}}^{n}\right)-p_{2} \varphi^{n}(t, x)+f_{1}(t, x)\right] \in L^{p}(Q)$,

$g_{3}=\left(\lambda_{n}-\lambda\right)\left[-g\left(t, x, \bar{\varphi}^{n}\right)+w(t, x)\right] \in L^{p}(\Sigma)$,

and so we get

$$
\begin{aligned}
\| u^{n, \lambda_{n}}- & u^{n, \lambda}\left\|_{W_{p}^{1,2}(Q)}+\right\| \zeta^{n, \lambda_{n}}-\zeta^{n, \lambda} \|_{W_{p}^{1,2}(\Sigma)} \\
\leq & C\left|\lambda_{n}-\lambda\right|\left\{\left\|\left[\Phi\left(t, x, \varphi_{x}^{n}\right) \frac{\partial}{\partial \varphi_{x_{j}}^{n}}\left(\Psi\left(t, x, \varphi_{x}^{n}\right) \varphi_{x_{i}}^{n}\right)-\delta_{i}^{j}\right] u_{x_{i} x_{j}}^{n, \lambda_{n}}\right\|_{L^{p}(Q)}\right. \\
& +\left\|u_{0}\right\|_{W_{\infty}^{2-\frac{2}{p}}(\Omega)}+\left\|\zeta_{0}\right\|_{W_{\infty}^{2-\frac{2}{p}}(\Gamma)}+\left\|A\left(t, x, \varphi_{x_{i}}^{n}\right)\right\|_{L^{p}(Q)}+p_{2}\left\|\varphi^{n}\right\|_{L^{p}(\Omega)} \\
& \left.+\left\|f_{1}\right\|_{L^{p}(Q)}+\left\|g\left(t, x, \bar{\varphi}^{n}\right)\right\|_{L^{p}(\Sigma)}+\|w\|_{W_{p}^{1-\frac{1}{2 p}, 2-\frac{1}{p}}(\Sigma)}\right\}
\end{aligned}
$$

for a positive constant $C\left(|\Omega|,|\Gamma|, p_{1}, p_{2}, M, M_{0}, M_{1}, M_{2}, M_{3}\right)$. By virtue of $\mathrm{I}_{1}, \mathrm{I}_{2}$ and knowing that $u_{x_{i} x_{j}}^{n, \lambda_{n}} \in L^{p}(Q)$, we derive the boundedness (in $L^{p}(Q)$ ) of the terms $A\left(t, x, \varphi_{x_{i}}^{n}\right)$, $\left(\Phi\left(t, x, \varphi_{x}^{n}\right) \frac{\partial}{\partial \varphi_{x_{j}}^{n}}\left(\Psi\left(t, x, \varphi_{x}^{n}\right) \varphi_{x_{i}}^{n}\right)-\delta_{i}^{j}\right) u_{x_{i} x_{j}}^{n, \lambda_{n}}, \varphi^{n}$ and $f_{1}$. Also, the sequence $\bar{\varphi}^{n}$ is bounded in $L^{p^{\prime}}(\Sigma)$, so that by (1.3) and (1.4) we derive the boundedness of $g\left(t, x, \bar{\varphi}^{n}\right)$ in $L^{p}(\Sigma)$. Therefore, since $\lambda_{n} \rightarrow \lambda$, we obtain from the previous inequality

$$
\left\|u^{n, \lambda_{n}}-u^{n, \lambda}\right\|_{W_{p}^{1,2}(Q)}+\left\|\zeta^{n, \lambda_{n}}-\zeta^{n, \lambda}\right\|_{W_{p}^{1,2}(\Sigma)} \rightarrow 0 \text { for } n \rightarrow \infty .
$$


In order to evaluate the difference $H\left(v^{n}, \lambda\right)-H(v, \lambda)$, we use (3.20) and (3.21), so that

$$
\begin{cases}p_{1} \frac{\partial}{\partial t}\left(u^{n, \lambda}-u^{\lambda}\right)-\left[\lambda \Phi\left(t, x, \varphi_{x}^{n}\right) .\right. & \\ \left.\quad \frac{\partial}{\partial \varphi_{x_{j}}^{n}}\left(\Psi\left(t, x, \varphi_{x}^{n}\right) \varphi_{x_{i}}^{n}\right)+(1-\lambda) \delta_{i}^{j}\right]\left(u_{x_{i} x_{j}}^{n, \lambda}-u_{x_{i} x_{j}}^{\lambda}\right) & \\ =\lambda\left\{\left[\Phi\left(t, x, \varphi_{x}^{n}\right) \frac{\partial}{\partial \varphi_{x_{j}}^{n}}\left(\Psi\left(t, x, \varphi_{x}^{n}\right) \varphi_{x_{i}}^{n}\right)-\delta_{i}^{j}\right] u_{x_{i} x_{j}}^{n, \lambda}\right. & \\ \left.\quad+A\left(t, x, \varphi_{x_{i}}^{n}\right)-p_{2} \varphi^{n}(t, x)+f_{1}(t, x)\right\} & \text { in } Q \\ \left(u^{n, \lambda}-u^{\lambda}\right)(t, x)=\left(\zeta^{n, \lambda}-\zeta^{\lambda}\right)(t, x) & \text { on } \Sigma \\ \left(u^{n, \lambda}-u^{\lambda}\right)(0, x)=0 & \text { in } \Omega \\ \frac{\partial}{\partial \mathbf{n}}\left(u^{n, \lambda}-u^{\lambda}\right)+p_{1} \frac{\partial}{\partial t}\left(\zeta^{n, \lambda}-\zeta^{\lambda}\right) & \\ \quad-\Delta_{\Gamma}\left(\zeta^{n, \lambda}-\zeta^{\lambda}\right)+p_{3}\left(\zeta^{n, \lambda}-\zeta^{\lambda}\right)=\lambda\left[-g\left(t, x, \bar{\varphi}^{n}\right)+w(t, x)\right] & \text { on } \Sigma \\ \left(\zeta^{n, \lambda}-\zeta^{\lambda}\right)(0, x)=0 & \text { in } \Gamma .\end{cases}
$$

Applying Lemma 7.4 to the linear inhomogeneous problem (3.26) with

$$
\begin{aligned}
& f_{3}=\lambda\left[A\left(t, x, \varphi_{x_{i}}^{n}\right)-p_{2} \varphi^{n}(t, x)+f_{1}(t, x)\right] \in L^{p}(Q), \\
& g_{3}=\lambda\left[-g\left(t, x, \bar{\varphi}^{n}\right)+w(t, x)\right] \in L^{p}(\Sigma)
\end{aligned}
$$

and $u_{0}=\zeta_{0}=0, \lambda \in[0,1]$, we obtain

$$
\begin{aligned}
\| u^{n, \lambda}- & u^{\lambda}\left\|_{W_{p}^{1,2}(Q)}+\right\| \zeta^{n, \lambda}-\zeta^{\lambda} \|_{W_{p}^{1,2}(\Sigma)} \\
\leq & C\left\{\left\|\left[\Phi\left(t, x, \varphi_{x}^{n}\right) \frac{\partial}{\partial \varphi_{x_{j}}^{n}}\left(\Psi\left(t, x, \varphi_{x}^{n}\right) \varphi_{x_{i}}^{n}\right)-\delta_{i}^{j}\right] u_{x_{i} x_{j}}^{n, \lambda}\right\|_{L^{p}(Q)}\right. \\
& +\left\|A\left(t, x, \varphi_{x_{i}}^{n}\right)\right\|_{L^{p}(Q)}+p_{2}\left\|\varphi^{n}\right\|_{L^{p}(\Omega)} \\
& \left.+\left\|f_{1}\right\|_{L^{p}(Q)}+\left\|g\left(t, x, \bar{\varphi}^{n}\right)\right\|_{L^{p}(\Sigma)}+\|w\|_{W_{p}^{1-\frac{1}{2 p}, 2-\frac{1}{p}}(\Sigma)}\right\}
\end{aligned}
$$

for a positive constant $C$. Then, the convergences: $\varphi^{n} \rightarrow \varphi$ in $W_{p}^{0,1}(Q), \bar{\varphi}^{n} \rightarrow \bar{\varphi}$ in $L^{p^{\prime}}(\Sigma)$, the continuity of the Nemytskij operator (see, [2] and references therein) and the boundedness of the terms in right-hand side of above inequality helps us to conclude that

$$
\left\|u^{n, \lambda}-u^{\lambda}\right\|_{W_{p}^{1,2}(Q)}+\left\|\zeta^{n, \lambda}-\zeta^{\lambda}\right\|_{W_{p}^{1,2}(\Sigma)} \rightarrow 0 \text { for } n \rightarrow \infty
$$

Making use of the relations (3.25) and (3.27), we derive the continuity of the nonlinear operator $H$ defined by (3.20). Moreover, the mapping $H$ is compact, what can easily be seen by writting it as the composition

$$
B \times[0,1] \rightarrow W_{p}^{1,2}(Q) \times W_{p}^{1,2}(\Sigma) \hookrightarrow B=W_{p}^{0,1}(Q) \times L^{p^{\prime}}(\Sigma),
$$

where the second map is an compact inclusion due to Lions-Peeter embedding theorem (see [5, p. 21]).

\section{THE REgUlaRity OF THE SOlUtiON $u(t, x)$}

Now we establish the existence of a number $\delta>0$ such that (see (3.20))

$$
(u, \zeta, \lambda) \in B \times[0,1] \text { with }(u, \zeta)=H(u, \zeta, \lambda) \Longrightarrow\|(u, \zeta)\|_{B}<\delta .
$$


The equality $(u, \zeta)=H(u, \zeta, \lambda)$ in (4.28) is equivalent to (see (1.1), (2.10) and (3.21))

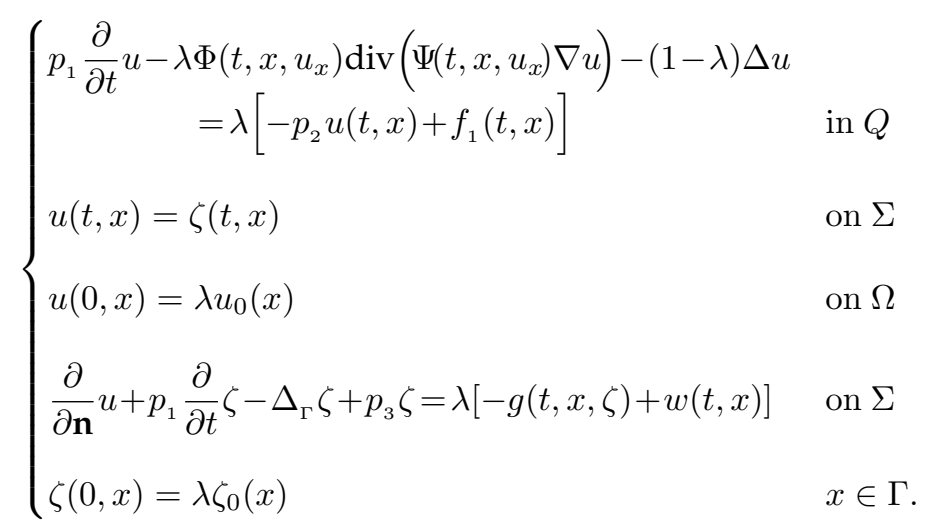

Multiplying (4.29) $)_{1}$ by $|u|^{p-2} u$ and integrating over $Q_{t}:=(0, t) \times \Omega, t \in(0, T]$, we get

$$
\begin{aligned}
& \frac{p_{1}}{p} \int_{O_{t}} \frac{\partial}{\partial t}|u(t, x)|^{p} d \tau d x \\
& \quad-\lambda \int_{Q_{t}} \Phi\left(t, x, u_{x}\right) \operatorname{div}\left(\Psi\left(t, x, u_{x}\right) \nabla u\right)|u|^{p-2} u d \tau d x \\
& \quad-(1-\lambda) \int_{Q_{t}} \Delta u|u|^{p-2} u d \tau d x \\
& \quad=\lambda p_{2} \int_{Q_{t}}|u|^{p} d \tau d x+\lambda \int_{Q_{t}} f_{1}|u|^{p-2} u d \tau d x
\end{aligned}
$$

In order to process the terms

$$
\int_{Q_{t}} \Phi\left(t, x, u_{x}\right) \operatorname{div}\left(\Psi\left(t, x, u_{x}\right) \nabla u\right)|u|^{p-2} u d \tau d x \text { and } \int_{Q_{t}} \Delta u|u|^{p-2} u d \tau d x,
$$

we use Green's first identity $(2.17)_{1}$ and $(2.17)_{2}$, respectively, and we obtain

$$
\begin{aligned}
& -\lambda \int_{Q_{t}} \Phi\left(t, x, u_{x}\right) \operatorname{div}\left(\Psi\left(t, x, u_{x}\right) \nabla u\right)|u|^{p-2} u d \tau d x \\
& =\lambda \int_{Q_{t}} \Psi\left(t, x, u_{x}\right) \nabla u \cdot \nabla\left(\Phi\left(t, x, u_{x}\right)|u|^{p-2} u\right) d \tau d x \\
& \quad+\lambda \int_{\Sigma_{t}} \Phi\left(t, x, u_{x}\right)|u|^{p-2} u\left(-\frac{\partial}{\partial \mathbf{n}} u\right) d \tau d \gamma, \\
& -(1-\lambda) \int_{Q_{t}} \Delta u|u|^{p-2} u d \tau d x=(1-\lambda)(p-1) \int_{Q_{t}}|\nabla u|^{2}|u|^{p-2} d \tau d x \\
& +(1-\lambda) \int_{\Sigma_{t}}|u|^{p-2} u\left(-\frac{\partial}{\partial \mathbf{n}} u\right) d \tau d \gamma,
\end{aligned}
$$

where $\Sigma_{t}=(0, t) \times \partial \Omega, t \in(0, T]$ and (see $\left.(4.29)_{4}\right)$.

$$
-\frac{\partial}{\partial \mathbf{n}} u=p_{1} \frac{\partial}{\partial t} \zeta-\Delta_{\Gamma} \zeta+p_{3} \zeta+\lambda g(t, x, \zeta)-\lambda w(t, x)
$$


Combining the above equality with the boundary condition (4.29) $)_{2}$ and, making use of the left inequality in (2.11), the hypothesis $\mathbf{I}_{2}, \mathbf{g}_{3}$, as well as the relations (4.31), (4.32), then (4.30) leads us to the following inequality

$$
\begin{aligned}
\frac{p_{1}}{p} \int_{\Omega} \mid & \left.u(t, x)\right|^{p} d x \\
+ & \lambda \frac{p_{1}}{p} m_{1} \int_{\Gamma}|\zeta(t, x)|^{p} d \gamma+(1-\lambda) \frac{p_{1}}{p} \int_{\Gamma}|\zeta(t, x)|^{p} d \gamma \\
& +\lambda \int_{Q_{t}} \Psi\left(t, x, u_{x}\right) \nabla u \cdot \nabla\left(\Phi\left(t, x, u_{x}\right)|u|^{p-2} u\right) d \tau d x \\
& +(1-\lambda)(p-1) \int_{Q_{t}}|\nabla u|^{2}|u|^{p-2} d \tau d x \\
& +\lambda p_{3} m_{1} \int_{\Sigma_{t}}|u|^{p} d \tau d \gamma+(1-\lambda) p_{3} \int_{\Sigma_{t}}|u|^{p} d \tau d \gamma \\
& +\lambda \int_{\Sigma_{t}} \nabla_{\Gamma}\left(\Phi\left(t, x, u_{x}\right)|u|^{p-1}\right) \cdot \nabla_{\Gamma} u d \tau d \gamma \\
& +(1-\lambda) \int_{\Sigma_{t}} \nabla_{\Gamma}\left(|u|^{p-1}\right) \cdot \nabla_{\Gamma} u d \tau d \gamma+\lambda b_{3} \int_{\Sigma_{t}}|u|^{p} d \tau d \gamma \\
\leq & \lambda \frac{p_{1}}{p} \int_{\Omega}\left|u_{0}(x)\right|^{p} d x+\lambda \frac{p_{1}}{p} m_{1} \int_{\Gamma}\left|\zeta_{0}(x)\right|^{p} d \gamma \\
& +(1-\lambda) \frac{p_{1}}{p} \int_{\Gamma}\left|\zeta_{0}(x)\right|^{p} d \gamma+\lambda p_{2} \int_{Q_{t}}|u|^{p} d \tau d x \\
& +\lambda \int_{Q_{t}} f_{1}|u|^{p-2} u d \tau d x+\lambda\left[(1-\lambda)+\lambda M_{1} \int_{\Sigma_{t}} w|u|^{p-2} u d \tau d \gamma\right.
\end{aligned}
$$

for all $t \in(0, T]$. The Hölder's and Cauchy's inequality, applied to the last terms in above inequality, give us

$$
\begin{gathered}
\mathbf{j}_{1} \cdot \lambda \int_{Q_{t}} f_{1}|u|^{p-2} u d \tau d x \leq \frac{p-1}{p} \int_{Q_{t}}|u|^{p} d \tau d x+\lambda \frac{1}{p} \int_{Q_{t}}\left|f_{1}\right|^{p} d \tau d x, \\
\mathbf{j}_{2} \cdot \lambda M_{1} \int_{\Sigma_{t}} w|u|^{p-2} u d \tau d \gamma \\
\leq \lambda \frac{p-1}{p} \int_{\Sigma_{t}}|u|^{p} d \tau d \gamma+M_{1} \frac{1}{p} \int_{\Sigma_{t}}|w|^{p} d \tau d \gamma,
\end{gathered}
$$

$\mathbf{j}_{3} \cdot(1-\lambda) \int_{\Sigma_{t}} w|u|^{p-2} u d \tau d \gamma$

$$
\leq(1-\lambda) \frac{p-1}{p} \int_{\Sigma_{t}}|u|^{p} d \tau d \gamma+\frac{1}{p} \int_{\Sigma_{t}}|w|^{p} d \tau d \gamma .
$$


Due to the inequalities $\mathbf{j}_{1}-\mathbf{j}_{3}$, from (4.33) we derive the following estimate

$$
\begin{aligned}
\frac{p_{1}}{p} \int_{\Omega} \mid & \left.u(t, x)\right|^{p} d x \\
+ & \lambda \frac{p_{1}}{p} m_{1} \int_{\Gamma}|\zeta(t, x)|^{p} d \gamma+(1-\lambda) \frac{p_{1}}{p} \int_{\Gamma}|\zeta(t, x)|^{p} d \gamma \\
& +\lambda \int_{Q_{t}} \Psi\left(t, x, u_{x}\right) \nabla u \cdot \nabla\left(\Phi\left(t, x, u_{x}\right)|u|^{p-2} u\right) d \tau d x \\
& +(1-\lambda)(p-1) \int_{Q_{t}}|\nabla u|^{2}|u|^{p-2} d \tau d x \\
& +\lambda p_{3} m_{1} \int_{\Sigma_{t}}|u|^{p} d \tau d \gamma+(1-\lambda) p_{3} \int_{\Sigma_{t}}|u|^{p} d \tau d \gamma \\
& +\lambda \int_{\Sigma_{t}} \nabla_{\Gamma}\left(\Phi\left(t, x, u_{x}\right)|u|^{p-1}\right) \cdot \nabla_{\Gamma} u d \tau d \gamma \\
& +(1-\lambda) \int_{\Sigma_{t}} \nabla_{\Gamma}\left(|u|^{p-1}\right) \cdot \nabla_{\Gamma} u d \tau d \gamma+\lambda b_{3} \int_{\Sigma_{t}}|\zeta|^{p} d \tau d \gamma \\
\leq & \lambda \frac{p_{1}}{p} \int_{\Omega}\left|u_{0}(x)\right|^{p} d x+\lambda \frac{p_{1}}{p} m_{1} \int_{\Gamma}\left|\zeta_{0}(x)\right|^{p} d \gamma \\
& +(1-\lambda) \frac{p_{1}}{p} \int_{\Gamma}\left|\zeta_{0}(x)\right|^{p} d \gamma+\left[p_{2}+2 \frac{p-1}{p}\right] \int_{Q_{t}}|u|^{p} d \tau d x \\
& +\lambda \frac{1}{p} \int_{Q_{t}}\left|f_{1}\right|^{p} d \tau d x+\left(M_{1}+1\right) \frac{1}{p} \int_{\Sigma_{t}}|w|^{p} d \tau d \gamma
\end{aligned}
$$

for all $t \in(0, T]$.

By Gronwall's lemma, from (4.34) we get

$$
\lambda\|u\|_{L^{p}(Q)}^{p} \leq C_{1}\left(\left\|u_{0}\right\|_{L^{p}(\Omega)}^{p}+\left\|\zeta_{0}\right\|_{L^{p}(\Gamma)}^{p}+\left\|f_{1}\right\|_{L^{p}(Q)}^{p}+\|w\|_{L^{p}(\Sigma)}^{p}\right),
$$

for a constant $C_{1}=C\left(|\Omega|,|\Gamma|, T, p, n, p_{1}, p_{2}, p_{3}, m_{1}, M_{1}\right)>0$.

Owing to (4.35), we deduce from (4.34) that

$$
\lambda\|\zeta\|_{L^{p}(\Sigma)}^{p} \leq C_{2}\left(\left\|u_{0}\right\|_{L^{p}(\Omega)}^{p}+\left\|\zeta_{0}\right\|_{L^{p}(\Gamma)}^{p}+\left\|f_{1}\right\|_{L^{p}(Q)}^{p}+\|w\|_{L^{p}(\Sigma)}^{p}\right),
$$

where $C_{2}=C\left(|\Omega|,|\Gamma|, T, n, p, p_{1}, p_{2}, p_{3}, m_{1}, M_{1}, b_{3}\right)>0$ denotes a new positive constant.

Appling Lemma 7.4 to the linear inhomogeneous problem (4.29) with

$$
\begin{aligned}
& f_{3}=\lambda\left[-p_{2} u(t, x)+f_{1}(t, x)\right] \in L^{p}(Q) \text { and } \\
& g_{3}=\lambda[-g(t, x, \zeta)+w(t, x)] \in L^{p}(\Sigma),
\end{aligned}
$$

we obtain

$$
\begin{aligned}
\|u\|_{W_{p}^{1,2}(Q)}+\|\zeta\|_{W_{p}^{1,2}(\Sigma)} & \\
\leq & C_{3}\left\{\left\|u_{0}\right\|_{W_{\infty}^{2-\frac{2}{p}}(\Omega)}+\left\|\zeta_{0}\right\|_{W_{\infty}^{2-\frac{2}{p}}(\Gamma)}+\lambda p_{2}\|u\|_{L^{p}(\Omega)}\right. \\
& \left.+\left\|f_{1}\right\|_{L^{p}(Q)}+\lambda\|g(t, x, \zeta)\|_{L^{p}(\Sigma)}+\|w\|_{W_{p}^{1-\frac{1}{2 p}, 2-\frac{1}{p}}(\Sigma)}\right\}
\end{aligned}
$$

for a constant $C_{3}=C\left(|\Omega|,|\Gamma|, T, n, p, p_{1}, p_{3}\right)>0$. 
Using now (1.4), then (4.37) becomes

$$
\begin{aligned}
\mid u\left\|_{W_{p}^{1,2}(Q)}+\right\| \zeta \|_{W_{p}^{1,2}(\Sigma)} \leq & C_{4}\left\{1+\left\|u_{0}\right\|_{W_{\infty}^{2-\frac{2}{p}}(\Omega)}+\left\|\zeta_{0}\right\|_{W_{\infty}^{2-\frac{2}{p}}(\Gamma)}\right. \\
& +\lambda\|u\|_{L^{p}(\Omega)}+\lambda\|\zeta\|_{L^{p^{\prime}}(\Sigma)} \\
& \left.+\left\|f_{1}\right\|_{L^{p}(Q)}+\|w\|_{W_{p}^{1-\frac{1}{2 p}, 2-\frac{1}{p}}(\Sigma)}\right\},
\end{aligned}
$$

for a constant $C_{4}=C\left(|\Omega|,|\Gamma|, T, n, p, p_{1}, p_{2}, p_{3}, m_{1}, M_{1}, b_{3}\right)>0$.

Owing to the embeddings in (3.23) 2 a standard interpolation inequality (see [5, p. 58]) yields that $\forall \epsilon>0, \exists C(\epsilon)>0$ such that

$$
\|\zeta\|_{L^{p^{\prime}}(\Sigma)} \leq \epsilon\|\zeta\|_{W_{p}^{1,2}(\Sigma)}+C(\epsilon)\|\zeta\|_{L^{p}(\Sigma)}
$$

and thus from (4.38), we get

$$
\begin{aligned}
\mid u \|_{W_{p}^{1,2}(Q)}+ & \left(1-\epsilon C_{4}\right)\|\zeta\|_{W_{p}^{1,2}(\Sigma)} \\
& \leq C_{5}\left\{1+\left\|u_{0}\right\|_{W_{\infty}^{2-\frac{2}{p}}(\Omega)}+\left\|\zeta_{0}\right\|_{W_{\infty}^{2-\frac{2}{p}}(\Gamma)}\right. \\
& +\lambda\|u\|_{L^{p}(\Omega)}+\lambda\|\zeta\|_{L^{p}(\Sigma)} \\
& \left.+\left\|f_{1}\right\|_{L^{p}(Q)}+\|w\|_{W_{p}^{1-\frac{1}{2 p}, 2-\frac{1}{p}}(\Sigma)}\right\}
\end{aligned}
$$

for a new constant $C_{5}=C(\epsilon) C_{4}>0$.

The continuous embedding in (3.23) ensures that

$$
\|u\|_{L^{p}(Q)}+\|\zeta\|_{L^{p}(\Sigma)} \leq C\left(\|u\|_{W_{p}^{1,2}(Q)}+\|\zeta\|_{W_{p}^{1,2}(\Sigma)}\right)
$$

which, for $\epsilon>0$ with $1-\epsilon C_{4}>0$, and thanks to (4.35)-(4.36) and (4.39), ensures that a constant $\delta>0$ can be found such that the property expressed in (4.28) is true.

Denoting $B_{\delta}^{H}:=\left\{(u, \zeta) \in B:\|(u, \zeta)\|_{B}<\delta\right\}$, relation (4.28) implies that

$$
(u, \zeta, \lambda) \neq(u, \zeta) \quad \forall(u, \zeta) \in \partial B_{\delta}^{H}, \forall \lambda \in[0,1],
$$

provided that $\delta>0$ is sufficiently large. Furthermore, following the same reasoning as in [2], we conclude that problem (2.10) has a solution $(u, \zeta) \in W_{p}^{1,2}(Q) \times W_{p}^{1,2}(\Sigma)$ (for more details, see [12, p. 195]). Estimate (2.13) follows from (4.39) combined with (4.35)-(4.36). This completes the proof of the first part in Theorem 2.1.

\section{PROOF OF Theorem 2.1 CONTINUED}

In this section we prove the second part of Theorem 2.1 which comes down to checking the estimate (2.16) and, as a consequence, the uniqueness of the solution to problem (1.1) (or (1.5)). To this end we consider $\left(u^{1}, \zeta^{1}\right),\left(u^{2}, \zeta^{2}\right)$ as in the statement of Theorem 2.1. In the first part we already esteblished that $u^{1}, u^{2} \in W_{p}^{1,2}(Q)$ and $\zeta^{1}, \zeta^{2} \in W_{p}^{1,2}(\Sigma)$. Thus $U=u^{1}-u^{2} \in W_{p}^{1,2}(Q)$ and $Z=\zeta^{1}-\zeta^{2} \in W_{p}^{1,2}(\Sigma)$.

Following [1, p. 176], we write the increments of $a_{i j}$ and $A$ (see (1.6)) in the form

$$
a_{i j}\left(t, x, u_{x}^{1}\right)-a_{i j}\left(t, x, u_{x}^{2}\right)=\int_{0}^{1} \frac{d}{d \lambda} a_{i, j}\left(t, x, u_{x}^{\lambda}\right) d \lambda,
$$




$$
A\left(t, x, u_{x}^{1}\right)-A\left(t, x, u_{x}^{2}\right)=\int_{0}^{1} \frac{d}{d \lambda} A\left(t, x, u_{x}^{\lambda}\right) d \lambda
$$

and then

$$
a_{i j}\left(t, x, u_{x}^{1}\right) u_{x_{i} x_{j}}^{1}-a_{i j}\left(t, x, u_{x}^{2}\right) u_{x_{i} x_{j}}^{2}=a_{i j}\left(t, x, u_{x}^{1}\right) U_{x_{i} x_{j}}
$$

$$
+\left\{u_{x_{i} x_{j}}^{2} \int_{0}^{1} \frac{\partial}{\partial u_{x_{j}}^{\lambda}}\left[\Phi\left(t, x, u_{x}^{\lambda}\right) a_{i, j}\left(t, x, u_{x}^{\lambda}\right)\right] d \lambda\right\} U_{x_{i}},
$$

$$
A\left(t, x, u_{x}^{1}\right)-A\left(t, x, u_{x}^{2}\right)=\left\{\int_{0}^{1} \frac{\partial}{\partial u_{x_{j}}^{\lambda}} A\left(t, x, u_{x}^{\lambda}\right) d \lambda\right\} U_{x_{i}},
$$

where

$$
\begin{aligned}
& a_{i, j}\left(t, x, u_{x}^{\lambda}\right)=\frac{\partial}{\partial u_{x_{j}}^{\lambda}}\left[\Psi\left(t, x, u_{x}^{\lambda}\right) u_{x_{i}}^{\lambda}\right], \\
& A\left(t, x, u_{x}^{\lambda}\right)=\Phi\left(t, x, u_{x}^{\lambda}\right) a_{i}\left(t, x, u_{x}^{\lambda}\right), a_{i}\left(t, x, u_{x}^{\lambda}\right)=\frac{\partial}{\partial x_{i}}\left[\Psi\left(t, x, u_{x}^{\lambda}\right) u_{x_{i}}^{\lambda}\right] \text { and } \\
& u_{x}^{\lambda}(t, x)=\lambda u_{x}^{1}(t, x)+(1-\lambda) u_{x}^{2}(t, x) .
\end{aligned}
$$

We subtract the equation (1.5) for $u^{2}(t, x)$ from the equations (1.5) for $u^{1}(t, x)$ and, owing to (5.40) and (5.41), we obtain the following linear problem endowed with nonlinear dynamic boundary conditions, that is

$$
\begin{cases}p_{1} \frac{\partial}{\partial t} U-\hat{a}_{i j}(t, x) \Delta U=-\hat{a}_{i}(t, x) \nabla U-p_{2} U+\left(f_{1}^{1}-f_{1}^{2}\right) & \text { in } Q \\ U(t, x)=Z(t, x) & \text { on } \Sigma \\ U(0, x)=\left(u_{0}^{1}-u_{0}^{2}\right)(x) & \text { in } \Omega \\ \frac{\partial}{\partial \mathbf{n}} U+p_{1} \frac{\partial}{\partial t} Z-\Delta_{\Gamma} Z+p_{3} Z & \text { on } \Sigma \\ =-\left[g\left(\zeta^{1}\right)-g\left(\zeta^{2}\right)\right]+\left(w^{1}-w^{2}\right) & \text { on } \Gamma\end{cases}
$$

where

$$
\begin{aligned}
\hat{a}_{i j}(t, x)= & \Phi\left(t, x, u_{x}^{1}\right) a_{i j}\left(t, x, u_{x}^{1}\right), \\
\hat{a}_{i}(t, x)= & -u_{x_{i} x_{j}}^{2} \int_{0}^{1} \frac{\partial}{\partial u_{x_{j}}^{\lambda}}\left[\Phi\left(t, x, u_{x}^{\lambda}\right) a_{i, j}\left(t, x, u_{x}^{\lambda}\right)\right] d \lambda \\
& +\int_{0}^{1} \frac{\partial}{\partial u_{x_{j}}^{\lambda}}\left[\Phi\left(t, x, u_{x}^{\lambda}\right) \frac{\partial}{\partial x_{i}} \Psi\left(t, x, u_{x}^{\lambda}\right) u_{x_{i}}^{\lambda}\right] d \lambda .
\end{aligned}
$$

By hypothesis we have $u_{0}^{1}-u_{0}^{2} \in W_{\infty}^{2-\frac{2}{p}}(\Omega) \subset W_{p}^{2-\frac{2}{p}}(\Omega), \zeta_{0}^{1}-\zeta_{0}^{2} \in W_{\infty}^{2-\frac{2}{p}}(\Gamma) \subset W_{p}^{2-\frac{2}{p}}(\Gamma)$, $-\hat{a}_{i}(t, x) \nabla U-p_{2} U+\left(f_{1}^{1}-f_{1}^{2}\right) \in L^{p}(Q)$ (recall that $\left.U(t, x) \in W_{p}^{1,2}(Q)\right)$ and $-\left[g\left(\zeta^{1}\right)-\right.$ 
$\left.g\left(\zeta^{2}\right)\right] \in L^{p}(\Sigma)$. So, Theorem 2.1 in [2, relation (2.7)] applied to problem (5.42) for the unknown functions $U(t, x)=\left(u^{1}-u^{2}\right)(t, x)$ and $Z(t, x)=\left(\zeta^{1}-\zeta^{2}\right)(t, x)$, gives the estimate

$$
\begin{aligned}
\| u^{1}- & u^{2}\left\|_{W_{p}^{1,2}(Q)}^{p}+\right\| \zeta^{1}-\zeta^{2} \|_{W_{p}^{1,2}(\Sigma)}^{p} \\
\leq & C_{6}\left[\left\|u_{0}^{1}-u_{0}^{2}\right\|_{W_{\infty}^{2-\frac{2}{p}}(\Omega)}^{p}+\left\|\zeta_{0}^{1}-\zeta_{0}^{2}\right\|_{W_{\infty}^{2-\frac{2}{p}(\Gamma)}}^{p}\right. \\
& +\left\|\nabla\left(u^{1}-u^{2}\right)\right\|_{L^{p}(Q)}^{p}+\left\|u^{1}-u^{2}\right\|_{L^{p}(Q)}^{p}+\left\|g\left(\zeta^{1}\right)-g\left(\zeta^{2}\right)\right\|_{L^{p}(\Sigma)}^{p} \\
& \left.+\left\|f_{1}^{1}-f_{1}^{2}\right\|_{L^{p}(Q)}^{p}+\left\|w^{1}-w^{2}\right\|_{L^{p}(\Sigma)}^{p}\right] .
\end{aligned}
$$

where $C_{6}=C\left(|\Omega|,|\Gamma|, T, n, p, p_{1}, p_{2}, p_{3}\right)>0$. Appling again Theorem 2.1 in [2, relation (2.4)] and making use of the embedding $W_{p}^{1,2}(Q) \subset L^{p}(Q)$, we have

$$
\begin{aligned}
\left\|\nabla\left(u^{1}-u^{2}\right)\right\|_{L^{p}(Q)}^{p}+\left\|u^{1}-u^{2}\right\|_{L^{p}(Q)}^{p} & \\
\leq & \bar{C}\left[1+\left\|u_{0}^{1}-u_{0}^{2}\right\|_{W_{\infty}^{2-\frac{2}{p}}(\Omega)}^{p}+\left\|\zeta_{0}^{1}-\zeta_{0}^{2}\right\|_{W_{\infty}^{2-\frac{2}{p}}(\Gamma)}^{p}\right. \\
& \left.+\left\|f_{1}^{1}-f_{1}^{2}\right\|_{L^{p}(Q)}^{p}+\left\|w^{1}-w^{2}\right\|_{W_{p}^{1-\frac{1}{2 p}, 2-\frac{1}{p}}(\Sigma)}^{p}\right] .
\end{aligned}
$$

Let us now focus our attention on the term $\left\|g\left(\zeta^{1}\right)-g\left(\zeta^{2}\right)\right\|_{L^{p}(\Sigma)}^{p}$ from the right-hand side of (5.43). Firstly, we recall that is true the following sequence of embeddings (see [8, p. 103, relation (2.198)]):

$$
W_{p}^{1,2}(\Sigma) \subset L^{p^{\prime}}(\Sigma) \subset L^{\ell_{1}}(\Sigma) \subset L^{p}(\Sigma) \subset L^{2}(\Sigma) .
$$

From $\mathbf{g}_{2}$, Hölder's inequality, relations (2.15) and (5.45), we derive that

$$
\left\|g\left(\zeta^{1}\right)-g\left(\zeta^{2}\right)\right\|_{L^{p}(\Sigma)} \leq C_{7}\left\|\zeta^{1}-\zeta^{2}\right\|_{L^{\ell_{1}}(\Sigma)} .
$$

where $C_{7}=C\left(|\Omega|, T, p, b_{2}\right)\left(1+2 M_{5}^{2}\right)$. Using the embedding in (5.45), the standard interpolation inequalities (see [5, p. 58]) yield that $\forall \varepsilon>0, \exists C(\varepsilon)>0$ such that

$$
\|y\|_{L^{\ell_{1}(\Sigma)}} \leq \varepsilon\|y\|_{W_{p}^{1,2}(\Sigma)}+C(\varepsilon)\|y\|_{L^{p}(\Sigma)}, \quad \forall y \in W_{p}^{1,2}(\Sigma) .
$$

Combining (5.44), (5.46) and (5.47), estimate (5.43) leads to

$$
\begin{aligned}
& \left\|u^{1}-u^{2}\right\|_{W_{p}^{1,2}(Q)}^{p}+\left(1-\epsilon C_{7}\right)\left\|\zeta^{1}-\zeta^{2}\right\|_{W_{p}^{1,2}(\Sigma)}^{p} \\
& \leq C_{6}\left\{\left\|u_{0}^{1}-u_{0}^{2}\right\|_{W_{\infty}^{2-\frac{2}{p}}(\Omega)}^{p}+\left\|\zeta_{0}^{1}-\zeta_{0}^{2}\right\|_{W_{\infty}^{2-\frac{2}{p}}(\Gamma)}^{p}\right. \\
& C_{6} \bar{C}\left[1+\left\|u_{0}^{1}-u_{0}^{2}\right\|_{W_{\infty}^{2-\frac{2}{p}}(\Omega)}^{p}+\left\|\zeta_{0}^{1}-\zeta_{0}^{2}\right\|_{W_{\infty}^{2-\frac{2}{p}}(\Gamma)}^{p}\right. \\
& \left.+\left\|f_{1}^{1}-f_{1}^{2}\right\|_{L^{p}(Q)}^{p}+\left\|w^{1}-w^{2}\right\|_{W_{p}^{1-\frac{1}{2 p}, 2-\frac{1}{p}}(\Sigma)}^{p}\right] \\
& \left.+C_{8}\left\|\zeta^{1}-\zeta^{2}\right\|_{L^{p}(\Sigma)}+\left\|f_{1}^{1}-f_{1}^{2}\right\|_{L^{p}(Q)}^{p}+\left\|w^{1}-w^{2}\right\|_{L^{p}(\Sigma)}^{p}\right\} .
\end{aligned}
$$


where $C_{8}=C(\varepsilon) C_{7}>0$.

In order to handle the term $\left\|\zeta^{1}-\zeta^{2}\right\|_{L^{p}(\Sigma)}$, we rely on a priori estimates in $L^{p}(\Sigma)$. In this respect, we multiply (5.42) $)_{1}$ by $|U|^{p-2} U=\left|u^{1}-u^{2}\right|^{p-2}\left(u^{1}-u^{2}\right)$. Integrating over $Q_{t}$, $t \in(0, T]$ and using Green's first identity as well as the Cauchy-Schwarz inequality, we get

$$
\begin{aligned}
\frac{p_{1}}{p} \int_{O_{t}} \frac{\partial}{\partial t} \mid & \left.U(t, x)\right|^{p} d \tau d x \\
& +(p-1) \int_{Q_{t}}|\nabla U|^{2} \cdot \nabla\left(\hat{a}_{i j}(t, x)|U|^{p-2}\right) d \tau d x \\
& +\int_{\Sigma_{t}}|U|^{p-1}\left(-\frac{\partial}{\partial \mathbf{n}} U\right) d \tau d \gamma \\
\leq & \int_{Q_{t}}\left|\hat{a}_{i}(t, x)\right||\nabla U||U|^{p-1} d \tau d x \\
& +p_{2} \int_{Q_{t}}|U|^{p} d \tau d x+\lambda \int_{Q_{t}}\left|f_{1}^{1}-f_{1}^{2}\right||U|^{p-1} d \tau d x .
\end{aligned}
$$

Using the boundary conditions $(5.42)_{2},(5.42)_{4}$ and Hölder's inequality, then (5.49) becomes

$$
\begin{aligned}
\frac{p_{1}}{p} \int_{O_{t}} & \frac{\partial}{\partial t}|U(t, x)|^{p} d \tau d x+\frac{p_{1}}{p} \int_{\Sigma_{t}} \frac{\partial}{\partial t}|Z(t, x)|^{p} d \tau d \gamma \\
& +(p-1) \int_{Q_{t}}|\nabla U|^{2} \cdot \nabla\left(\hat{a}_{i j}(t, x)|U|^{p-2}\right) d \tau d x \\
& +p_{3} \int_{\Sigma_{t}}|Z(t, x)|^{p} d \tau d \gamma+(p-1) \int_{\Sigma_{t}}\left|\nabla_{\Gamma} Z\right|^{2}|Z|^{p-2} d \tau d \gamma \\
& +\int_{\Sigma_{t}}\left[g\left(\zeta^{1}\right)-g\left(\zeta^{2}\right)\right]|Z|^{p-2} Z d \tau d \gamma \\
\leq & \tilde{C}\left[\left\|\nabla\left(u^{1}-u^{2}\right)\right\|_{L^{p}(Q)}^{p}+\left\|u^{1}-u^{2}\right\|_{L^{p}(Q)}^{p}\right. \\
& +\| u^{1}-\left.u^{2}\right|_{L^{p}(Q)} ^{p}+\left|\zeta^{1}-\zeta^{2}\right|_{L^{p}(\Sigma)}^{p} \\
& \left.+\left\|f_{1}^{1}-f_{1}^{2}\right\|_{L^{p}(Q)}^{p}+\left\|w^{1}-w^{2}\right\|_{L^{p}(\Sigma)}^{p}\right]
\end{aligned}
$$

where $\tilde{C}=C\left(|\Omega|,|\Gamma|, p, p_{2}, M_{1}, M_{4}\right)>0$. 
In particular, owing to hypothesis $\mathbf{g}_{1}$ and (5.44), it follows from (5.50) that

$$
\begin{aligned}
\int_{\Omega} \mid u^{1}- & \left.u^{2}\right|^{p} d x+\int_{\Gamma}\left|\zeta^{1}-\zeta^{2}\right|^{p} d \gamma \\
\leq & C_{9}\left[\left\|u_{0}^{1}-u_{0}^{2}\right\|_{L^{p}(\Omega)}^{p}+\left\|\zeta_{0}^{1}-\zeta_{0}^{2}\right\|_{L^{p}(\Gamma)}^{p}\right. \\
& +\left\|f_{1}^{1}-f_{1}^{2}\right\|_{L^{p}(Q)}^{p}+\left\|w^{1}-w^{2}\right\|_{L^{p}(\Sigma)}^{p} \\
& \left.+\int_{0}^{t}\left[\int_{\Omega}\left|u^{1}-u^{2}\right|^{p} d x+\int_{\Gamma}\left|\zeta^{1}-\zeta^{2}\right|^{p} d \gamma\right] d \tau\right],
\end{aligned}
$$

where $C_{9}=C\left(\tilde{C}, p_{3}, b_{1}\right)>0$.

Making uses of Gronwall's inequality, we can deduce from (5.51) that

$$
\begin{aligned}
\left\|u^{1}-u^{2}\right\|_{L^{p}(Q)}^{p}+\left\|\zeta^{1}-\zeta^{2}\right\|_{L^{p}(\Sigma)}^{p} \\
\quad \leq \exp ^{C_{9} T}\left[\left\|u_{0}^{1}-u_{0}^{2}\right\|_{L^{p}(\Omega)}^{p}+\left\|\zeta_{0}^{1}-\zeta_{0}^{2}\right\|_{L^{p}(\Gamma)}^{p}\right. \\
\left.\quad+\left\|f_{1}^{1}-f_{1}^{2}\right\|_{L^{p}(Q)}^{p}+\left\|w^{1}-w^{2}\right\|_{L^{p}(\Sigma)}^{p}\right] .
\end{aligned}
$$

Making use of relations (5.52), from (5.48) we finally derive that

$$
\begin{gathered}
\left\|u^{1}-u^{2}\right\|_{W_{p}^{1,2}(Q)}+\left(1-\epsilon C_{7}\right)\left\|\zeta^{1}-\zeta^{2}\right\|_{W_{p}^{1,2}(\Sigma)} \\
\leq C_{10}\left[1+\left\|u_{0}^{1}-u_{0}^{2}\right\|_{W_{\infty}^{2-\frac{2}{p}}(\Omega)}+\left\|\zeta_{0}^{1}-\zeta_{0}^{2}\right\|_{W_{\infty}^{2-\frac{2}{p}}(\Gamma)}\right. \\
\left.+\left\|f_{1}^{1}-f_{1}^{2}\right\|_{L^{p}(Q)}+\left\|w^{1}-w^{2}\right\|_{W_{p}^{1-\frac{1}{2 p}, 2-\frac{1}{p}}(\Sigma)}\right] \\
+C_{8} \exp ^{C_{9} T}\left[\left\|u_{0}^{1}-u_{0}^{2}\right\|_{L^{p}(\Omega)}^{p}+\left\|\zeta_{0}^{1}-\zeta_{0}^{2}\right\|_{L^{p}(\Gamma)}^{p}\right. \\
\left.+\left\|f_{1}^{1}-f_{1}^{2}\right\|_{L^{p}(Q)}^{p}+\left\|w^{1}-w^{2}\right\|_{L^{p}(\Sigma)}^{p}\right] .
\end{gathered}
$$

For $\varepsilon>0$ with $1-\varepsilon C_{7}>0$, the embedding $W_{p}^{1-\frac{1}{2 p}, 2-\frac{1}{p}}(\Sigma) \subset L^{p}(\Sigma)$ and the estimate (5.53) implies the estimate (2.16), which finishes the proof of Theorem 2.1.

As a consequence, the uniqueness of solution to problem (1.1) is valid.

Corollary 5.1. For the same initial conditions, the problem (1.1) possesses a unique classical solution.

Proof. Let $f_{1}^{1}=f_{1}^{2}=f$ and $w^{1}=w^{2}=w$ in the Theorem 2.1. Then (2.16) demonstrates the corollary (see also [4, Theorem 2.4, p. 17]).

\section{CONCLUSIONS}

The problem addressed in this paper is a nonlinear second-order anisotropic reactiondiffusion equation with principal part in divergence form, endowed with nonlinear inhomogeneous dynamic boundary conditions and non-constant mobility $\Psi(t, x, \cdot)$. Provided 
that the initial and boundary data meet appropriate regularity and compatibility conditions, we prove the existence, estimate, uniqueness and regularity of a classical solution. Precisely, the Leray-Schauder principle is applied to prove the existence result for the nonlinear problem in question, while the $L^{p}$ theory of linear and quasi-linear parabolic equations, via Lemma $A_{3}$ in Appendix, is involved in order to derive regularity properties for the solutions. In other words, we can not directly apply the $L^{p}$ theory to the problem (1.1) or (1.5). Thus, it makes the result in Lemma $\mathbf{7 . 4}$ himself very important. Moreover, the a priori estimates are made in $L^{p}(Q)$ and $L^{p}(\Sigma)$ which leads to a better estimates for unknown functions $(u(t, x), \zeta(t, x))$. This approach could be applied in future to study other kind of the first and second boundary value problems.

From the perspective of applicability, it is natural to find the suitable type of nonlinearities on the $\partial \Omega$, able to describe the complexity of many important physical phenomena, among which we mention effect of surface tension, separating zone of solid and liquid states etc. So, one of the most important characteristics of our improved mathematical model (1.1) is the nonlinear term $g$ in the nonlinear dynamic boundary conditions which allows to consider a nonlinearity with a larger growth exponent $r^{\prime} \leq(n+2) /(n+2-2 p)$ if $n+2>2 p$ (see (3.19). It extends the already studied types of boundary conditions (see [1], [7]) and therefore makes the new formulation of model (1.1) to be more able to describe a wide variety of industrial applications, in particular, the interactions with the walls in confined systems (i.e. the phase changes at the boundary of $\Omega$ ).

Let's also remark that, due to the presence of the term $\Phi\left(t, x, u_{x}\right)$, the nonlinear operator in $(1.1)_{1}$ does not represent the gradient of the energy functional. Therefore, the new proposed second-order nonlinear problem can not be obtained from the minimisation of any energy cost functional, i.e. (1.1) is not a variational PDE model.

The qualitative results obtained here can be involved later in the quantitative approaches of the mathematical model (1.1) as well as in the study of distributed and/or boundary nonlinear optimal control problems governed by such a nonlinear problem. Amongst other things, we wish to be exploited all this in our future works.

At the end we want to underline the solutions dependence in Theorem 2.1 on physical parameters, which can be useful in future investigations regarding the error analysis and numerical simulations.

\section{APPENDIX}

The goal of this section is to analyze some simple problems which are useful in the proof of Theorem 2.1. Every product is understood in the $L^{2}$-space, except when otherwise specified. In particular, the norm and the scalar product in $L^{2}(\Omega)$ are denoted by $\|\cdot\|$ and $\langle.,$.$\rangle , respectively, while the corresponding symbols in L^{2}(\Gamma)$ will be marked by a subscript $\Gamma$.

Consider the family of linear first-order boundary value problems with homogeneous Dirichlet conditions

$$
\begin{cases}p_{1} \frac{\partial}{\partial t} v-\lambda \Phi\left(t, x, v_{x}\right) \operatorname{div}\left(\Psi\left(t, x, v_{x}\right) \nabla v(t, x)\right)-(1-\lambda) \Delta v(t, x)=f_{2}(t, x) & \text { in } Q \\ v(t, x)=0 & \text { on } \Sigma \\ v(0, x)=v_{0}(x) & \text { in } \Omega\end{cases}
$$

where $\lambda \in[0,1]$.

Let us now formulate the existence result for the classical solution of problems (7.54). 
Lemma 7.2. Suppose $v(t, x)$ is a classical solution of problem (7.54) and that the conditions in [1, Theorem 2.1, p.173] hold.

Then, $\forall v_{0}(x) \in W_{p}^{2-\frac{2}{p}}(\Omega)$ and $\forall f_{2}(t, x) \in L^{p}(Q)$, with $p \neq \frac{3}{2}$, the problem (7.54) has a solution $v(t, x) \in W_{p}^{1,2}(Q)$ and the next estimate holds

$$
\|v\|_{W_{p}^{1,2}(Q)} \leq C\left[\left\|v_{0}\right\|_{W_{p}^{2-\frac{2}{p}}(\Omega)}+\left\|f_{2}\right\|_{L^{p}(Q)}\right],
$$

where the constant $C$ depends on $|\Omega|, T, n, p, p_{1}$, but is independent of $v$ and $f_{2}$.

Proof. The result established by Lemma 7.2 was proved in [1] in a more general context (i.e., in the presence of the reaction term and inhomogeneous boundary conditions of the Cauchy-Neumann type). We omit details here. For similar results involving other classes of functions for existence, we recommend to readers the reference $[4$, Chapter V, Section 6, p. 449].

Next, we consider the family of linear first-order boundary value problem with inhomogeneous Dirichlet condition and zero initial datum, that is

$$
\begin{cases}p_{1} \frac{\partial}{\partial t} v-\lambda \Phi\left(t, x, v_{x}\right) \operatorname{div}\left(\Psi\left(t, x, v_{x}\right) \nabla v(t, x)\right)-(1-\lambda) \Delta v(t, x)=0 & \text { in } Q \\ v(t, x)=y(t, x) & \text { on } \Sigma \\ v(0, x)=0 & \text { in } \Omega,\end{cases}
$$

where $\lambda \in[0,1]$.

Lemma 7.3. If $y \in W_{p}^{1-\frac{1}{2 p}, 2-\frac{1}{p}}(\Sigma)$, then there exists a unique solution $v \in W_{p}^{1,2}(Q)$ to problem (7.56) such that

$$
\|v\|_{W_{p}^{1,2}(Q)} \leq C\|y\|_{W_{p}^{1-\frac{1}{2 p}, 2-\frac{1}{p}}(\Sigma)},
$$

where $C$ depends on $|\Gamma|, T, n, p, p_{1}$, but is independent of $v$ and $y$.

Moreover

$$
\int_{0}^{t}\left\langle\frac{\partial}{\partial \mathbf{n}} v(\tau, \cdot), y(\tau, \cdot)\right\rangle_{\Gamma} d \tau \geq 0 .
$$

Proof. We consider the linear extension operator

$$
T_{0}: W_{p}^{1-\frac{1}{2 p}, 2-\frac{1}{p}}(\Sigma) \rightarrow W_{p}^{1,2}(Q) \quad \text { such that }\left.\left(T_{0} y\right)\right|_{\Sigma}=y .
$$

(see [2] and references therein). Besides that, let us consider the change of variable $z=v-T_{0} y$. Knowing that $v=z+T_{0} y$ solves (7.56), i.e.

$$
\begin{cases}p_{1} \frac{\partial}{\partial t}\left(z+T_{0} y\right)-\lambda \Phi\left(t, x, v_{x}\right) \operatorname{div}\left(\Psi\left(t, x, v_{x}\right) \nabla\left(z+T_{0} y\right)\right) & \\ -(1-\lambda) \Delta\left(z+T_{0} y\right)=0 & \text { in } Q \\ z+T_{0} y=y & \text { on } \Sigma \\ \left(z+T_{0} y\right)(0, x)=0 & \text { in } \Omega,\end{cases}
$$

and making use of the last statement in (7.59), we obtain that $z$ in (7.60) solves (7.54) with 


$$
f_{2}(t, x)=\frac{\partial}{\partial t}\left(T_{0} y\right)+\Phi\left(t, x, v_{x}\right) \operatorname{div}\left(\Psi\left(t, x, v_{x}\right) \nabla\left(T_{0} y\right)\right)+(1-\lambda) \Delta\left(T_{0} y\right) \in L^{p}(Q)
$$

and $v_{0}(x)=-T_{0} y(0, x) \in W_{p}^{2-\frac{2}{p}}(\Gamma)$. Applying Lemma 7.2 we derive the existence and uniqueness of the solution to problem (7.56) as well as the estimate (7.57).

Multiplying equation (7.56) by $v$ and then integrating over $(0, t) \times \Omega$, we get

$$
\begin{aligned}
& \lambda \int_{0}^{t} \Phi\left(t, x, v_{x}\right)\left\langle\frac{\partial}{\partial \mathbf{n}} v(\tau, \cdot), y(\tau, \cdot)\right\rangle_{\Gamma} d \tau+(1-\lambda) \int_{0}^{t}\left\langle\frac{\partial}{\partial \mathbf{n}} v(\tau, \cdot), y(\tau, \cdot)\right\rangle_{\Gamma} d \tau \\
& \quad=\frac{p_{1}}{2}\|v(t)\|^{2} \\
& \quad+\lambda \int_{0}^{t}\left\langle\Psi\left(\tau, \cdot, v_{x}(\tau, \cdot)\right) \nabla v(\tau, \cdot), \nabla\left(\Phi\left(\tau, \cdot, v_{x}(\tau, \cdot)\right) v(\tau, \cdot)\right)\right\rangle_{\Omega} d \tau \\
& \quad+(1-\lambda) \int_{0}^{t}\|\nabla v(\tau)\|^{2} d \tau \geq 0
\end{aligned}
$$

from which we can deduce (7.58) and thus Lemma 7.3 is proved.

We now analyze the linear inhomogeneous problem with linear homogeneous dynamic boundary condition, that is

$$
\begin{cases}p_{1} \frac{\partial}{\partial t} v-\lambda \Phi\left(t, x, v_{x}\right) \operatorname{div}\left(\Psi\left(t, x, v_{x}\right) \nabla v\right)-(1-\lambda) \Delta v=f_{3} & \text { in } Q \\ v=y & \text { on } \Sigma \\ v(0, x)=v_{0}(x) & x \in \Omega \\ \frac{\partial}{\partial \mathbf{n}} v+p_{1} \frac{\partial}{\partial t} y-\Delta_{\Gamma} y+p_{3} y+g_{3}(t, x)=0 & \text { on } \Sigma \\ y(0, x)=y_{0}(x) & x \in \Gamma .\end{cases}
$$

Lemma 7.4. If $v_{0} \in W_{p}^{2-\frac{2}{p}}(\Omega), y_{0} \in W_{p}^{2-\frac{2}{p}}(\Gamma), f_{3} \in L^{p}(Q), g_{3} \in L^{p}(\Sigma)$, then (7.61) possesses a unique solution $(v, y) \in W_{p}^{1,2}(Q) \times W_{p}^{1,2}(\Sigma)$ such that

$$
\|v\|_{W_{p}^{1,2}(Q)}+\|y\|_{W_{p}^{1,2}(\Sigma)}
$$

$$
\leq C\left[\left\|v_{0}\right\|_{W_{p}^{2-\frac{2}{p}}(\Omega)}+\left\|y_{0}\right\|_{W_{p}^{2-\frac{2}{p}}(\Gamma)}+\left\|f_{3}\right\|_{L^{p}(Q)}+\left\|g_{3}\right\|_{L^{p}(\Sigma)}\right],
$$

where $C$ depends on $|\Omega|,|\Gamma|, T, n, p, p_{1}, p_{3}$, but is independent of $v, y, f_{3}$ and $g_{3}$.

Proof. The proof is based on the idea used in [9, Lemma 2.2]. Following this, we have that there exists the operator $T_{0}: W_{p}^{1-\frac{1}{2 p}, 2-\frac{1}{p}}(\Sigma) \rightarrow W_{p}^{1,2}(Q)$ (see (7.59)), defined as the unique solution $v \in W_{p}^{1,2}(Q)$ to the following problem:

$$
\begin{cases}p_{1} \frac{\partial}{\partial t} v-\Phi\left(t, x, v_{x}\right) \operatorname{div}\left(\Psi\left(t, x, v_{x}\right) \nabla v(t, x)\right)=0 & \text { in } Q \\ v=y & \text { on } \Sigma \\ v(0, x)=0 & \text { in } \Omega .\end{cases}
$$


Setting $z(t)=T_{0} y(t)$ and $\theta(t)=v(t)-z(t)$, we derive from (7.59) and (7.61) that

$$
\begin{cases}p_{1} \frac{\partial}{\partial t} \theta-\Phi\left(t, x, \theta_{x}\right) \operatorname{div}\left(\Psi\left(t, x, \theta_{x}\right) \nabla \theta(t, x)\right)=f_{3}(t, x) & \text { in } Q \\ \theta=0 & \text { on } \Sigma \\ \theta(0, x)=v_{0}(x) & x \in \Omega \\ \frac{\partial}{\partial \mathbf{n}} v+p_{1} \frac{\partial}{\partial t} y-\Delta_{\Gamma} y+p_{3} y+g_{3}(t, x)=0 & \text { on } \Sigma \\ y(0, x)=y_{0}(x) & x \in \Gamma .\end{cases}
$$

Let us first observe that in this new setting of problem (7.61), the unknown functions $(\theta, y)$ are no longer coupled, which means that we can treat separately equations $(7.64)_{1-3}$ and $(7.64)_{4-5}$. Accordingly, Lemma 7.2 applied to problem $(7.64)_{1-3}$ gives the existence of a solution $\theta \in W_{p}^{1,2}(Q)$ which satisfies the estimate

$$
\|\theta\|_{W_{p}^{1,2}(Q)} \leq C\left[\left\|v_{0}\right\|_{W_{p}^{2-\frac{2}{p}}(\Omega)}+\left\|f_{3}\right\|_{L^{p}(Q)}\right],
$$

while the unknown $y$ in problem $(7.64)_{4-5}$ solves the problem (we recall that $v=\theta+z=$ $\left.\theta+T_{0} y\right)$

$$
\begin{cases}\frac{\partial}{\partial \mathbf{n}}\left(T_{0} y\right)+p_{1} \frac{\partial}{\partial t} y-\Delta_{\Gamma} y+p_{3} y+\bar{g}_{3}(t, x)=0 & \text { on } \Sigma \\ y(0, x)=y_{0}(x) & x \in \Gamma,\end{cases}
$$

where $\bar{g}_{3}(t, x)=g_{3}(t, x)+\frac{\partial}{\partial \mathbf{n}} \theta \in L^{p}(\Sigma)$. Moreover, due to Lemma 7.3 and to a suitable trace theorem, we have

$$
\left\|\frac{\partial}{\partial \mathbf{n}}\left(T_{0} y\right)\right\|_{L^{p}(\Sigma)} \leq C\|y\|_{W_{p}^{1-\frac{1}{2 p}, 2-\frac{1}{p}}(\Sigma)} .
$$

Arguing as in [9, p. 723] we obtain the existence and uniqueness of the solution $y$ solving (7.66), as well as the following estimate

$$
\|y\|_{W_{p}^{1,2}(\Sigma)} \leq C\left[\left\|y_{0}\right\|_{W_{p}^{2-\frac{2}{p}}(\Gamma)}+\|y\|_{W_{p}^{1-\frac{1}{2 p}, 2-\frac{1}{p}}(\Sigma)}+\left\|\bar{g}_{3}\right\|_{L^{p}(\Sigma)}\right] .
$$

A standard interpolation inequality (see [5, p. 58]), written for the Sobolev spaces $W_{p}^{1,2}(\Sigma) \subset W_{p}^{1-\frac{1}{2 p}, 2-\frac{1}{p}}(\Sigma) \subset L^{2}(\Sigma)$, yields that $\forall \varepsilon>0, \exists C(\varepsilon)>0$ such that

$$
\|y\|_{W_{p}^{1-\frac{1}{2 p}, 2-\frac{1}{p}(\Sigma)}} \leq \varepsilon\|y\|_{W_{p}^{1,2}(\Sigma)}+C(\varepsilon)\|y\|_{L^{2}(\Sigma)} .
$$

Applying (7.69) in order to handle the second term in the right-hand side of (7.68), we obtain

$$
(1-\varepsilon)\|y\|_{W_{p}^{1,2}(\Sigma)} \leq C(\varepsilon)\left[\left\|y_{0}\right\|_{W_{p}^{2-\frac{2}{p}}(\Gamma)}+\|y\|_{L^{2}(\Sigma)}+\left\|\bar{g}_{3}\right\|_{L^{p}(\Sigma)}\right] .
$$

Multiplying (7.66) by $y(t)$, scalarly in $L^{2}(\Gamma)$, Integrating over $[0, t]$ and using (7.58), we get

$$
\frac{1}{2}\|y(t)\|_{L^{2}(\Gamma)}^{2}-\frac{1}{2}\left\|y_{0}\right\|_{L^{2}(\Gamma)}^{2} \leq \int_{0}^{t}\left\langle\bar{g}_{3}(\tau), y(\tau)\right\rangle_{\Gamma} d \tau .
$$


By Gronwall's lemma, we obtain

$$
\|y\|_{L^{2}(\Sigma)} \leq C(T)\left(\left\|y_{0}\right\|_{L^{2}(\Gamma)}+\left\|\bar{g}_{3}\right\|_{L^{2}(\Sigma)}\right),
$$

which, combined with (7.65), (7.70) and making use of the embedding $W_{p}^{2-\frac{2}{p}}(\Gamma) \subset L^{2}(\Gamma)$, yields estimate (7.62) and finishes the proof of Lemma 7.4.

\section{REFERENCES}

[1] Barbu, T.; Miranville, A.; Moroşanu, C. A qualitative analysis and numerical simulations of a nonlinear second-order anisotropic diffusion problem with non-homogeneous Cauchy-Neumann boundary conditions. Applied Mathematics and Computation 350 (2019), 170-180, doi: 10.1016/j.amc.2019.01.004.

[2] Cârjă, O.; Miranville, A.; Moroşanu, C. On the existence, uniqueness and regularity of solutions to the phase-field system with a general regular potential and a general class of nonlinear and non-homogeneous boundary conditions. Nonlinear Analysis. TMA, 113 (2015), 190-208, http:/ /dx.doi.org/10.1016/j.na.2014.10.003.

[3] Gavriluţ, A.; Moroşanu, C., Well-Posedness for a Nonlinear Reaction - Diffusion Equation Endowed with Nonhomogeneous Cauchy- Neumann Boundary Conditions and Degenerate Mobility. ROMAI J. 14 (2018), no. 1, 129-141, https:/ / rj.romai.ro/arhiva/2018/1/Gavrilut-Morosanu-final.pdf.

[4] Ladyzhenskaya, O. A.; Solonnikov, B. A.; Uraltzava, N. N. Linear and quasi-linear equations of parabolic type. Prov. Amer. Math. Soc. 1968.

[5] Lions, J. L. Control of distributed singular systems. Gauthier-Villars, Paris, 1985.

[6] Miranville, A.; Moroşanu, C. On the existence, uniqueness and regularity of solutions to the phase-field transition system with non-homogeneous Cauchy-Neumann and nonlinear dynamic boundary conditions. Appl. Math. Modell. 40 (2016), no. 1, 192-207, doi: 10.1016/j.apm.2015.04.039.

[7] Miranville, A.; Moroşanu, C. A Qualitative Analysis of a Nonlinear Second-Order Anisotropic Diffusion Problem with Non-homogeneous Cauchy-Stefan-Boltzmann Boundary Conditions. Appl. Math. Optim. 84 (2021), no. 1, 227-244, doi:10.1007/s00245-019-09643-5.

[8] Miranville, A.; Moroşanu, C. Qualitative and Quantitative Analysis for the Mathematical Models of Phase Separation and Transition. Applications, American Institute of Mathematical Sciences, Differential Equations \& Dynamical Systems, 7 (2020), ISBN-10: 1-60133-024-3 ISBN-13: 978-1-60133-024-6 www.aimsciences.org/fileAIMS/cms/news/info/28df2b3d-ffac-4598-a89b-9494392d1394.pdf.

[9] Miranville, A.; Zelik, S. Exponential attractors for the Cahn-Hilliard equation with dynamic boundary conditions. Math Methods Appl Sci. 28 (2005), 709-735.

[10] Moroşanu, C. Analysis and optimal control of phase-field transition system: Fractional steps methods, Bentham Science Publishers, eBooks, 2012, http:/ /dx.doi.org/10.2174/97816080535061120101.

[11] Moroşanu, C. Qualitative and quantitative analysis for a nonlinear reaction-diffusion equation. ROMAI J. 12 (2016), no. 2, 85-113, https:/ / rj.romai.ro/arhiva/2016/2/Morosanu.pdf.

[12] Moroşanu, C. Well-posedness for a phase-field transition system endowed with a polynomial nonlinearity and a general class of nonlinear dynamic boundary conditions. J. Fixed Point Theory Appl. 18 (2016), 225-250, doi: $10.1007 /$ s11784-015-0274-8.

[13] Moroşanu, C.; Motreanu, D. The phase field system with a general nonlinearity. Int. J. Differ. Equ. Appl. 1 (2000), no. 2, 187-204.

TiRASPOL STATE UNIVERSITY, REPUBlic OF MOLDOVA

INSTITUTE OF MATHEMATICS AND COMPUTER SCIENCES OF ASM

CHIŞINĂU, REPUBLIC OF MOLDOVA

Email address: mmchoban@gmail. com

ALEXANDRU IOAN CUZA UNIVERSITY OF IASI - UAIC

IAŞI, BD. CAROL I, NO. 11, 700506, ROMANIA

Email address: costica.morosanu@uaic.ro 